\title{
Aerodynamic Performance and Vibration Analyses of Small Scale Horizontal Axis Wind Turbine with Various Number of Blades
}

\author{
Mosfequr Rahman', Emile Maroha1, Adel El Shahat' ${ }^{2}$, Valentin Soloiu', Marcel Ilie ${ }^{1}$ \\ ${ }^{1}$ Mechanical Engineering Department, Georgia Southern University, Statesboro, USA \\ ${ }^{2}$ Electrical Engineering Department, Georgia Southern University, Statesboro, USA \\ Email:mrahman@georgiasouthern.edu,emilemaroha@gmail.com, aahmed@georgiasouthern.edu, \\ vsoloiu@georgiasouthern.edu,milie@georgiasouthern.edu
}

How to cite this paper: Rahman, M., Maroha, E., El Shahat, A., Soloiu, V. and Ilie, M. (2018) Aerodynamic Performance and Vibration Analyses of Small Scale Horizontal Axis Wind Turbine with Various Number of Blades. Journal of Power and Energy Engineering, 6, 76-105.

https://doi.org/10.4236/jpee.2018.66006

Received: November 27, 2017

Accepted: June 25, 2018

Published: June 28, 2018

Copyright $\odot 2018$ by authors and Scientific Research Publishing Inc. This work is licensed under the Creative Commons Attribution International License (CC BY 4.0).

http://creativecommons.org/licenses/by/4.0/

\begin{abstract}
The need to generate power from renewable sources to reduce demand for fossil fuels and the damage of their resulting carbon dioxide emissions is now well understood. Wind is among the most popular and fastest growing sources of alternative energy in the world. It is an inexhaustible, indigenous resource, pollution-free, and available almost any time of the day, especially in coastal regions. As a sustainable energy resource, electrical power generation from the wind is increasingly important in national and international energy policy in response to climate change. Experts predict that, with proper development, wind energy can meet up to $20 \%$ of US needs. Horizontal Axis Wind Turbines (HAWTs) are the most popular because of their higher efficiency. The aerodynamic characteristics and vibration of small scale HAWT with various numbers of blade designs have been investigated in this numerical study in order to improve its performance. SolidWorks was used for designing Computer Aided Design (CAD) models, and ANSYS software was used to study the dynamic flow around the turbine. Two, three, and five bladed HAWTs of $87 \mathrm{~cm}$ rotor diameter were designed. A HAWT tower of $100 \mathrm{~cm}$ long and $6 \mathrm{~cm}$ diameter was considered during this study while a shaft of $10.02 \mathrm{~cm}$ diameter was chosen. A good choice of airfoils and angle of attack is a key in the designing of a blade of rough surface and maintaining the maximum lift to drag ratio. The S818, S825 and S826 airfoils were used from the root to the tip and $4^{\circ}$ critical angle of attack was considered. In this paper, a more appropriate numerical models and an improved method have been adopted in comparable with other models and methods in the literature. The wind flow around the whole wind turbine and static behavior of the HAWT rotor was solved using Moving Reference Frame (MRF) solver. The HAWT
\end{abstract}


rotor results were used to initialize the Sliding Mesh Models (SMM) solver and study the dynamic behavior of HAWT rotor. The pressure and velocity contours on different blades surfaces were analyzed and presented in this work. The pressure and velocity contours around the entire turbine models were also analyzed. The power coefficient was calculated using the Tip Speed Ratio (TSR) and the moment coefficient and the results were compared to the theoretical and other research. The results show that the increase of number of blades from two to three increases the efficiency; however, the power coefficient remains relatively the same or sometimes decreases for five bladed turbine models. HAWT rotors and shaft vibrations were analyzed for two different materials using an applied pressure load imported from ANSYS fluent environment. It has proven that a good choice of material is crucial during the design process.

\section{Keywords}

Horizontal Axis Wind Turbine, Power Coefficient, Moment Coefficient, Mode Shape, Natural Frequency, CFD, Moving Reference Frame, Sliding Mesh Model

\section{Introduction}

With the global energy demand rising to unprecedented numbers, there is an obvious need to generate power from renewable sources to reduce the greenhouse gas emissions and achieve the U.S department of energy future goals of wind energy ( $20 \%$ wind energy by 2030 , and $35 \%$ by 2050 ) [1]. Renewable energy sources include wind energy, solar energy, tidal energy, geo-thermal energy, and biomass energy. Wind is among the most popular sources of alternative energy because it is pollution free and available almost any time of the day, especially in the coastal regions [2]. Winds are commonly classified by their spatial scale, their speed, the type of forces that cause them, the regions in which they occur, and their effect. Winds have various aspects, an important one being its velocity; other aspects are the density of the gas involved and the energy content or wind energy [3].

The two primary types of wind turbine are horizontal axis wind turbine (HAWT) and vertical axis wind turbine (VAWT) [4]. Horizontal axis means the rotating axis of the wind turbine is horizontal, or parallel to the ground. In the vertical axis wind turbine, the rotating axis of the wind turbine stands vertical or perpendicular to the ground. HAWTs are the most popular configuration because they have higher efficiency [5]. In a HAWT the generator converts directly the wind which is extracted from the rotor. HAWT are conventional wind turbines and unlikely the VAWT are not omnidirectional [5]. They must have some means for orienting the rotor with respect to the wind.

Leithead et al. [6] studied dynamics of variable speed wind turbines and design 
of models to control wind turbines. The purpose of this study was to investigate the dynamics of variable speed wind turbines and determine suitable models to support the control design task. Murtagh et al. [7] investigated control wind turbine vibration by incorporating a passive control device. A passive control method using a tuned mass damper to mitigate vibrations of the blades and tower of a wind turbine was introduced. Ashwani K. et al. have done structural and Modal analysis of Al 2024 wind turbine blade designed based on FEA [8]. Their analysis results show that the maximum deformation occurs at tip and the stresses are very less for $\mathrm{Al}$ lightweight materials.

Duque et al. [9] explored the ability of various methods to predict wind turbine power and aerodynamic loads. Results showed that all the methods, namely blade element momentum (BEM), vortex lattice, and Reynolds averaged Navier Stokes (RANS), perform well for pre-stall regimes. They found that the RANS code OVERFLOW, although not perfect, gives better predictions of power production for stall and post stall regime modeling than other methods. The combination of three methods has been used by [10] to test the performance of three HAWT blade shapes. They concluded that the wind tunnel test results show that the OPT and the UOT blades obtain the same maximum power coefficient $(\mathrm{Cp}=$ 0.428 ) but at different tip speed ratio points. The UUT blade obtains the lowest $\mathrm{Cp}$ value because it almost always operates in stall conditions.

D. S. Li et al. [11] performed the field experiment and numerical simulation methods to test the pressure distribution on a $33 \mathrm{KW} \mathrm{HAWT.} \mathrm{They} \mathrm{noticed} \mathrm{that}$ the closer to the root of blade the more consistent to the experimental results of the blade pressure. Ece Sagol et al. [12] performed the icing simulation method on wind turbine blades to the data representing the wind flow field. This method consists of creating a wind turbine model using Computer Aided Design (CAD) software followed by using ANSYS-FLUENT software to simulate the model. C. J. Bai et al. [13] designed a horizontal axis wind blade of 10,000 power output using the BEM and the modified stall model. The simulation results are compared with the improved BEM theory at rated wind speed of $10 \mathrm{~m} / \mathrm{s}$ and show that the CFD is a good method on aerodynamic investigation of a HAWT blade. The CFD method has been used by [14] to compute the aerodynamics of HAWT and a VAWT designs for low-cost rural applications. This method has been done in three different phases. In the first phase, a preliminary steady flow field is generated by rotating the turbine at a fixed rotation in the presence of the corresponding free stream wind speed.

\section{Goals and Objectives of the Research}

The goal of this numerical study is to analyze the designing of HAWT blade models to optimize wind turbine performance and vibrations in considering maximization of the power produced by a wind turbine and reduction of vibrations of the turbine's drive train and shaft. To achieve this goal, the following objectives were set for the present study: 
1) Design a HAWT's blade and entire turbine mechanical system using SolidWorks CAD software;

2) Design two, three, and five bladed HAWT rotor models with high lift force and low drag to minimize the blade's turbulence and vibration;

3) Analyze the rotor moment coefficients and power coefficients using numerical and theoretical method;

4) Compare the numerical results to the theoretical and other research;

5) Investigate the HAWT system vibration using ANSYS Fluent, Structural, and Modal environment.

\section{Turbine Modeling and Simulation}

Two, three, and five bladed HAWT rotors have been designed and numerically tested using SolidWorks and ANSYS FLUENT software. The aerodynamic performance results from ANSYS FLUENT simulations are presented in this study and com-pared with the theoretical results and other research.

Modal analysis is used to determine a structure's vibration characteristics including its natural frequency, mode shapes and mode participation factors (how much a mode participates in a given direction). Modal analysis is the most fundamental of the dynamic analysis and is used as the starting point for other dynamic analysis in ANSYS as it helps in calculating solution controls including step time. It allows the design to avoid resonant vibrations and gives an idea of how the model will respond to different types of dynamic loadings. Different materials are used to try to reduce the amount of vibration on the shaft and to find the optimum material and geometry that will cause the least amount of vibration.

In this research, entire HAWT and the turbine shaft are analyzed in ANSYS using modal analysis. HAWT and shaft's natural frequencies have been investigated for structural steel and aluminum alloy material and the results were compared to other research. The shaft natural frequencies at different mode shapes were calculated theoretically and the results were compared with the numerical results.

FEA study of two, three, and five HAWT rotors has also been done during this work to investigate the HAWT blades deflection frequencies and mode shapes. The procedure of importing the pressure distributions of the HAWT blade surfaces from ANSYS FLUENT to ANSYS Static structural, and then from ANSYS Static Structural to ANSYS Modal environment per-formed in this case is implemented.

\subsection{Blade, Shaft and Entire Turbine Model Design}

HAWT blade model was first designed using SolidWorks CAD software. The blade geometry of $45.25 \mathrm{~cm}$ long starts with a cylindrical shape at the root and then transitions to the airfoils S818, S825 and S826 for the root, body and tip, respectively. These airfoil shapes were chosen to provide an increasing blade 
width from the tip to the root, the blade tends to be thicker than the aerodynamic optimum close to the root, where the stresses due to bending are greatest. They can also contribute in the creation of blade surface roughness which facilitates the smooth wind flow around the blade surface. This blade was created by modifying the large-scale GE 1.5XLE turbine [14]. The blade made from aluminum and homogenized composite materials were considered during the analysis process. It has a varying thickness and it also has a spar inside the blade for structural rigidity. The blade needs to be designed with an increasing twist angle from the tip to the root because the closer to the tip of the blade the faster the blade is moving through the air and so the apparent wind angle is greater. Figure 1 shows an increasing twist angle from the tip to the root. The final blade geometry was then created using these airfoils and is shown in Figure 2.

A shaft of $20 \mathrm{~cm}$ long and three sections was then deigned, the shaft sections diameters are $0.5 \mathrm{inch}, 0.75 \mathrm{inch}$, and $1 \mathrm{inch}$ respectively from the tip to the root as shown in Figure 3. Two bearings are attached on the shaft.

The blade was then assembled with the hub and a circular pattern tool of

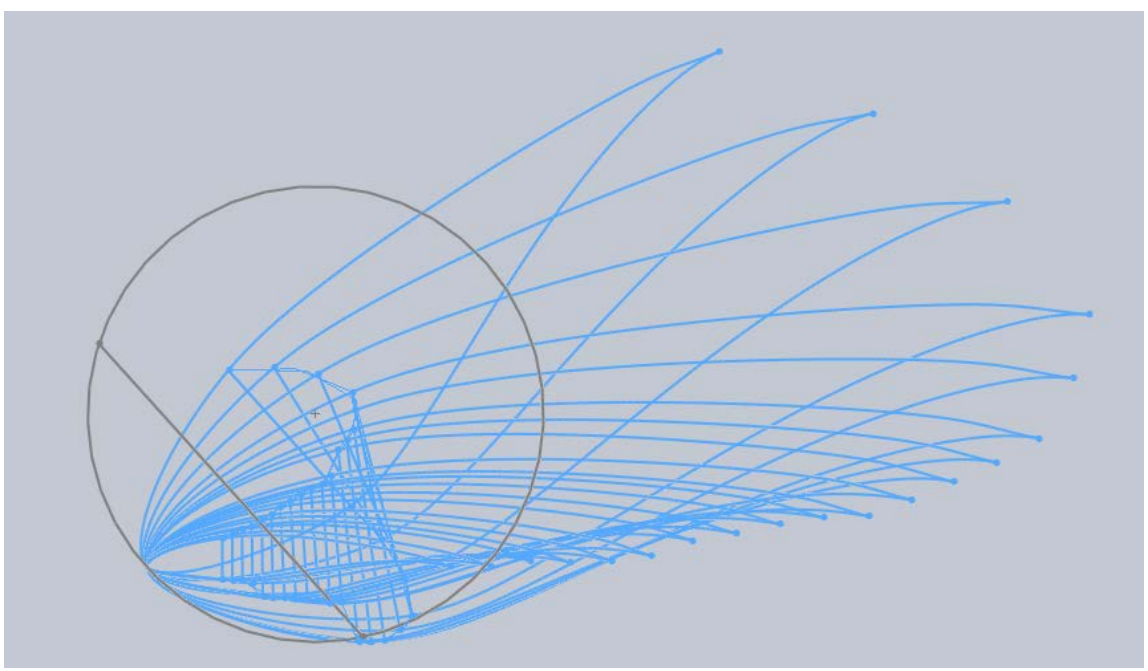

Figure 1. Increasing twist angle from the tip to the root.

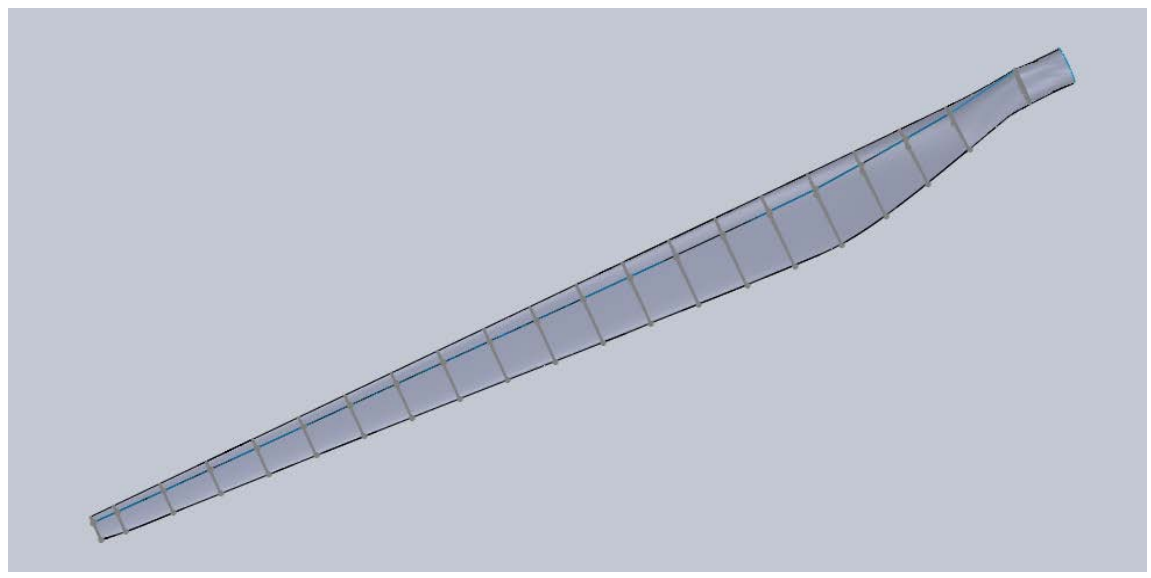

Figure 2. Solid works blade geometry. 


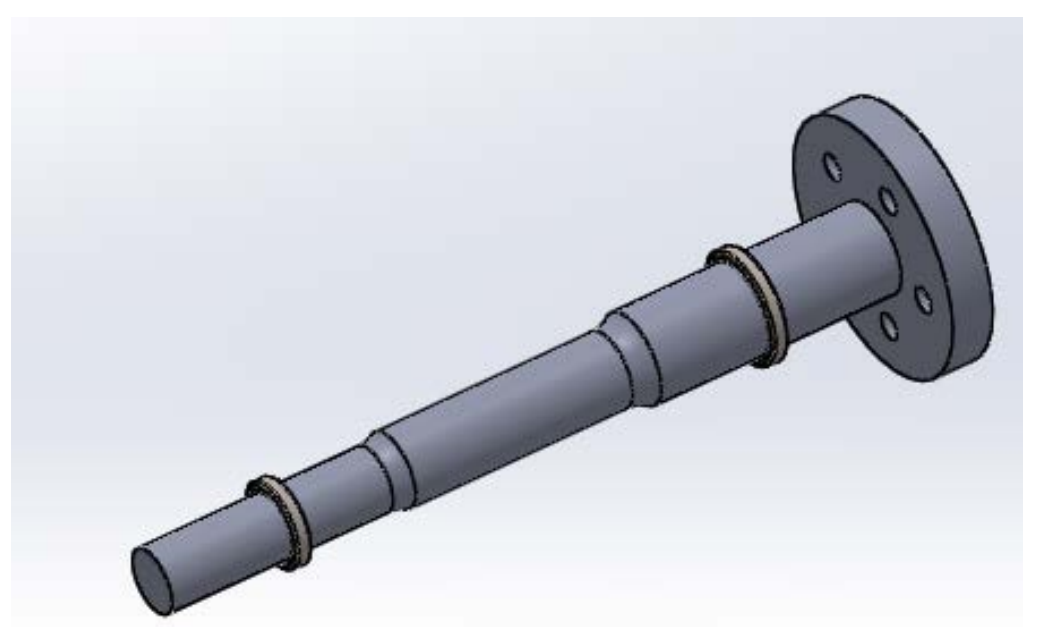

Figure 3. Shaft geometry.

SolidWorks CAD software was applied to create a three bladed HAWT rotor. The HAWT tower, rotor, shaft, nacelle, gear box, and generator were then assembled using Solid-Works CAD software to create the entire HAWT geometry shown in Figure 4. A tower of $100 \mathrm{~cm}$ long and $6 \mathrm{~cm}$ diameter was used during this study.

\subsection{Rotors Fluid Domains and Mesh}

The blade model was imported into ANSYS Design Modeler, and the two, three and five bladed HAWT rotors were created using circular pattern tool of ANSYS Design Modeler as shown in Figure 5.

Each rotor has a diameter of $87 \mathrm{~cm}$ and two fluid domains were then added to all geometries; one is a cylindrical fluid domain of $100 \mathrm{~cm}$ diameter rotating around $\mathrm{z}$-axis and is known as rotating domain. The second is a box fluid domain of $140 \mathrm{~cm}$ long and $60 \mathrm{~cm}$ width is known as stationary far-field fluid domain. The different computational domains are presented in Figure 6. The medium mesh of the model and fluid domains was generated and the number of nodes and elements are presented in Table 1 . The front view of medium mesh of each HAWT rotor model and fluid domains are shown in Figure 7.

\subsection{Entire Fluid Domains and Mesh}

The entire HAWT was imported into ANSYS Design Modeler and the wind flow analysis around the whole wind turbine was performed using CFD technique. The HAWT geometry is shown in Figure 8 .

As mentioned earlier, the first step is to study the wind flow around the whole HAWT, the pressure distributions on the HAWT surfaces will be imported into ANSYS structural environment for farther analyses. The HAWT was enclosed in the far field stationary fluid domain of $140 \mathrm{~cm}$ long, $50 \mathrm{~cm}$ width, and $200 \mathrm{~cm}$ high. The medium mesh of the computational fluid do-main was generated and is shown in Figure 9 as well as the number of nodes and elements. 


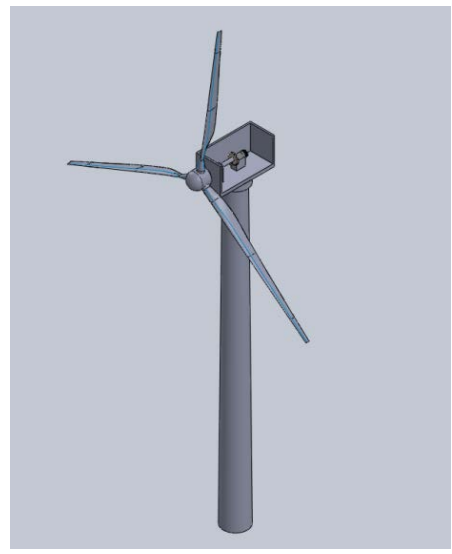

Figure 4. Entire HAWT geometry.
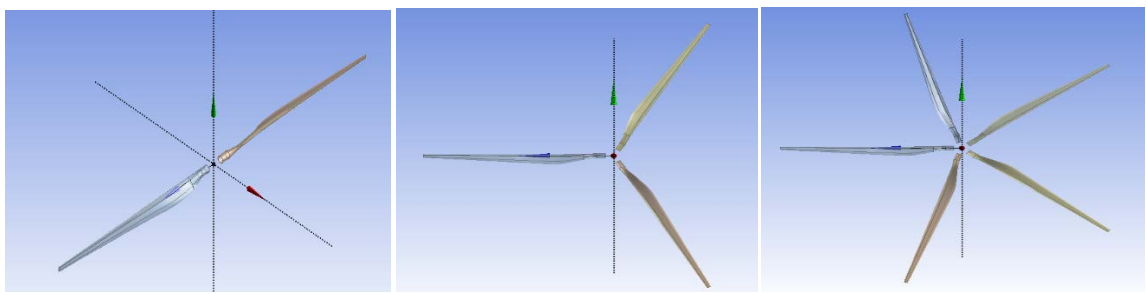

Figure 5. HAWT rotors geometries in design modeler of ANSYS.

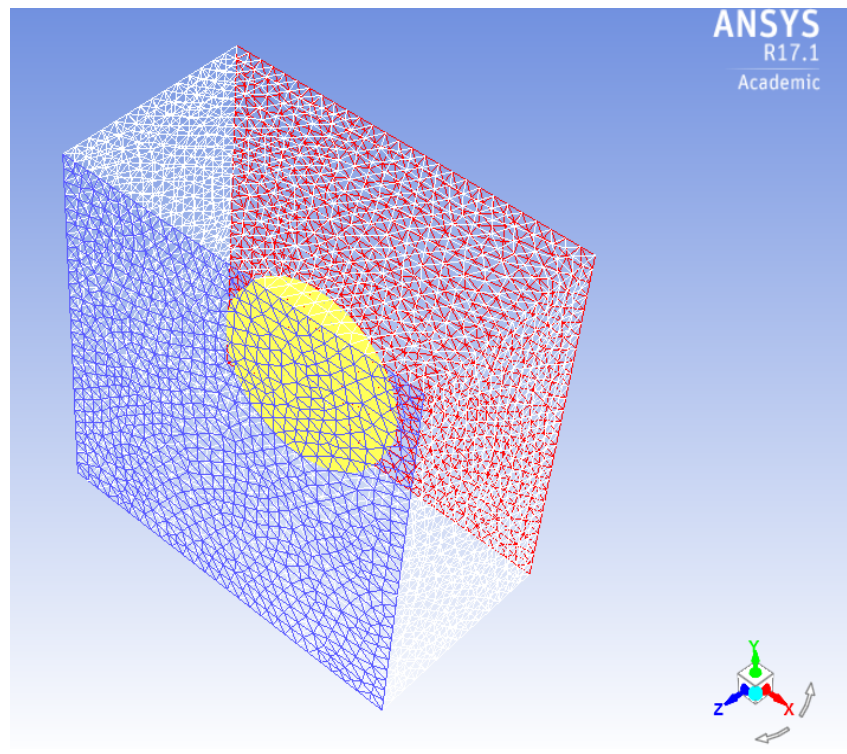

Figure 6. Computational domains.

\subsection{Computational Fluid Dynamic (CFD) Method}

The shear stress transport (SST) K-turbulence model was used for each model to allow a use of two equation model to ac-count for history effects like convection and diffusion of turbulent energy, as well as the eddy viscosity model commonly called the turbulent viscosity. The following are the governing equations for the SST K-turbulence models [15]:

- Turbulence kinetic energy 


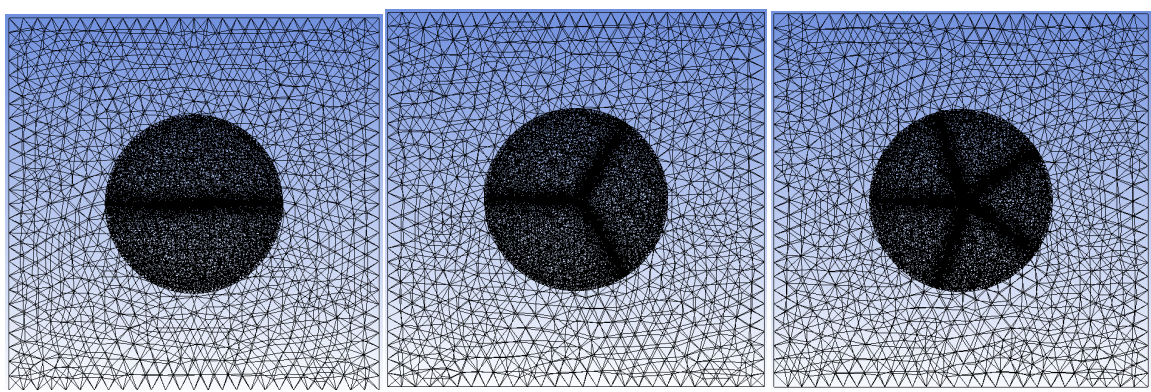

Figure 7. Mesh of two, three and five bladed HAWT rotors and their fluid domains.

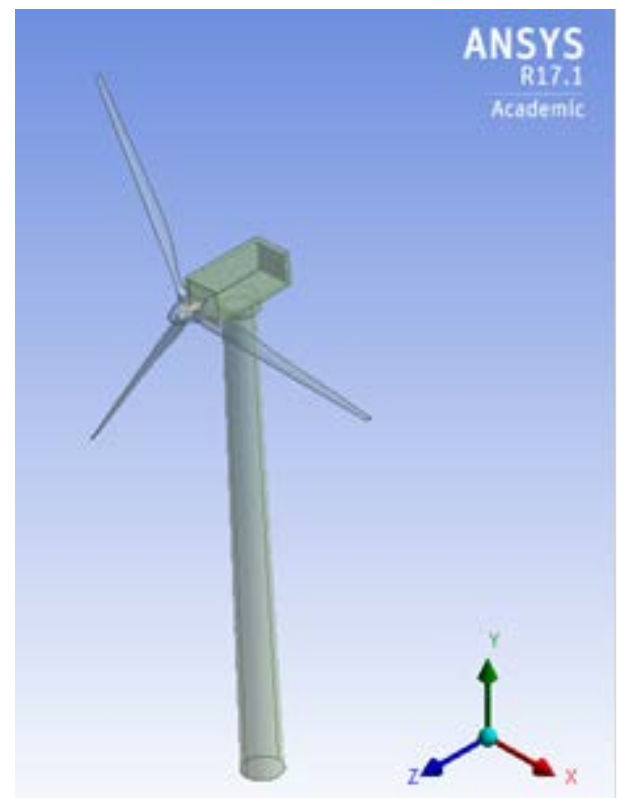

Figure 8. HAWT geometry in design modeler of ANSYS.

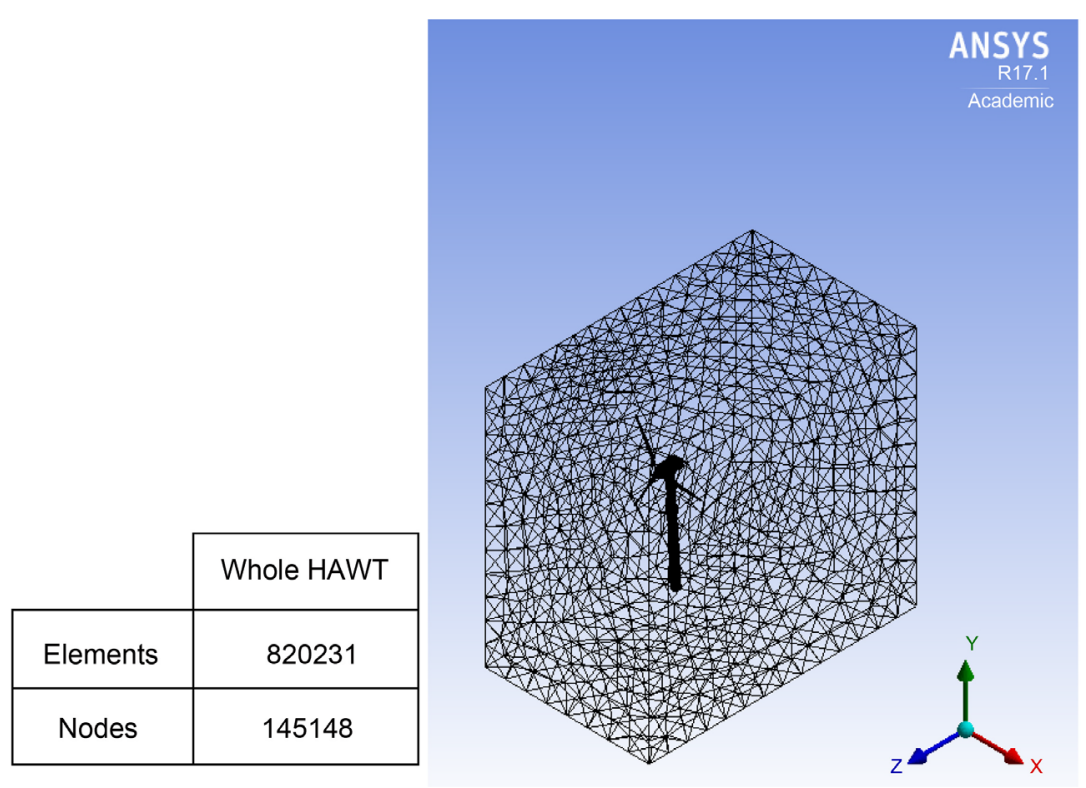

Figure 9. Mesh of whole HAWT and its fluid domain. 
Table 1. HAWT rotors and fluid domains number of element and nodes.

\begin{tabular}{cccc}
\hline & Two bladed HAWT & Three bladed HAWT & Five bladed HAWT \\
\hline Number of elements & 408,831 & 441,697 & 770,230 \\
Number of nodes & 93,194 & 101,567 & 183,935 \\
\hline
\end{tabular}

$$
\frac{\partial k}{\partial t}+U_{j} \frac{\partial k}{\partial x_{j}}=P_{k}-\beta^{*} k \omega+\frac{\partial}{\partial x_{j}}\left[\left(v+\sigma_{k} v_{T}\right) \frac{\partial k}{\partial x_{j}}\right]
$$

- Specific dissipation rate

$$
\frac{\partial \omega}{\partial t}+U_{j} \frac{\partial \omega}{\partial x_{j}}=\alpha S^{2}-\beta \omega^{2}+\frac{\partial}{\partial x_{j}}\left[\left(v+\sigma_{\omega} v_{T}\right) \frac{\partial \omega}{\partial x_{j}}\right]+2\left(1-F_{1}\right) \sigma_{\omega^{2}} \frac{1}{\omega} \frac{\partial k}{\partial x_{i}} \frac{\partial \omega}{\partial x_{i}}
$$

- Kinematic eddy viscosity

$$
v_{t}=\frac{\alpha_{1} k}{\max \left(\alpha_{1} \omega, S F_{2}\right)}
$$

where $P_{k}, F_{1}$, and $F_{2}$ are production limit, first and second bending functions respectively.

The rotor computational domain consists of a rotating zone surrounding the blades and a stationary far-field zone. A mesh interface was created between the two zones. The interface was necessary because the nodes on the boundaries of the far-field and rotational zones were intentionally non-conformal. The interface paired these so that interpolation can occur and fluid may pass into the rotating region. For each case, a static simulation with moving reference frame (MRF) and a dynamic sliding mesh model (SMM) were performed. The rotation was first defined using the steady-state solver with MRF, and the simulation was then solved in a transient manner using a sliding mesh motion. The converged static result from the MRF simulation was used to initialize the transient SMM solver. The moment Coefficient $(\mathrm{Cm})$ was monitored over time with accurate reference values for one full rotation. At 477 RPM, one rotation is completed in $0.1258 \mathrm{sec}$. A time step of $0.001747 \mathrm{sec}$ was chosen so that the $\mathrm{Cm}$ was calculated for every 5 degrees of rotation. This resulted in 72 time steps per simulation and 60 iterations per time step. From the $\mathrm{Cm}$ data, the power coefficient $(\mathrm{Cp})$ of the wind turbine can be easily calculated. Boundary conditions for the simulations included air velocity inlet, rotational speed of the blades, and pressure outlet. The rotating zone was set to 477 RPM for each simulation. The blade walls inside the rotating domain were given a no slip condition. Constant wind speeds along the $\mathrm{z}$ direction were set at the velocity inlet. Five different wind speeds of $4,6,8,10$, and $12 \mathrm{~m} / \mathrm{s}$ were tested for each model. The pressure outlet was kept at constant atmospheric pressure.

The optimum angle of attack must be maintained during wind turbine operation to maximize the blade efficiency and protect the turbine from vibration due to drag. The lift to drag ratio as function of angle of attack have been investigated during this work using ANSYS FLUENT software and the results are presented in 
Table 2. It was notice the current blade design has an optimum angle of attack of 4 degree. The plot of lift to drag ratio is shown in Figure 10, which shows that a peak of lift to drag ratio of $47 \%$ occurs at 4 -degree angle of attack which corresponds to the optimum angle of attack of the blade design. This optimum angle of attack was considered during the HAWT blades simulation process.

\subsection{Mathematical Equations for Theoretical Calculation}

The power generated by a cylindrical column of free air moving at a constant speed $V$ is given Equation (4).

$$
P=\frac{\mathrm{d} E}{\mathrm{~d} t}
$$

where $E$ is the kinetic energy given by:

$$
E=\frac{1}{2} m V^{2}
$$

Substituting Equation (5) into Equation (4), we get:

$$
P=\frac{\mathrm{d}\left(\frac{1}{2} m V^{2}\right)}{\mathrm{d} t}
$$

For constant wind speed,

Table 2. Lift to drag ratio as function of angle of attack.

\begin{tabular}{cl}
\hline Angle of attack (degree) & $\mathrm{C}_{\mathrm{l}} / \mathrm{C}_{\mathrm{d}}$ \\
\hline 0 & 0.05 \\
2 & 0.04 \\
4 & 0.47 \\
6 & 0.11 \\
8 & 0.08 \\
10 & 0.11 \\
12 & 0.11 \\
14 & 0.16 \\
\hline
\end{tabular}

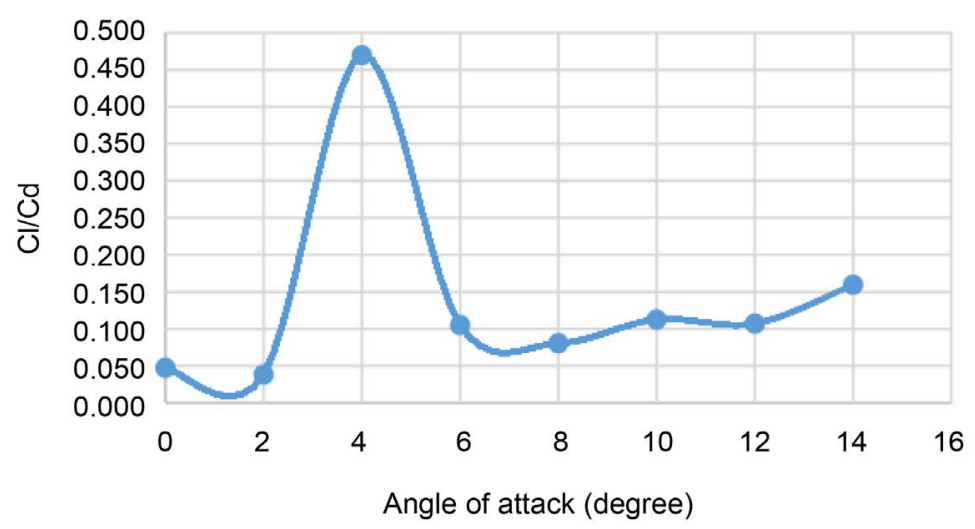

Figure 10. Lift to drag ratio vs angle of attack. 


$$
\frac{\mathrm{d} V}{\mathrm{~d} t}=0
$$

Consequently, Equation (6) becomes

$$
P=\frac{1}{2} \dot{m} V^{2}
$$

If the cross-sectional area of the column of air is $A$, and its density is $\rho$, the mass flow rate is given by:

$$
\dot{m}=\rho A V
$$

By substituting Equation (9) into Equation (8), we get:

$$
P=\frac{1}{2} \rho A V^{3}
$$

If the diameter of the column of air is $D$, then

$$
P=\frac{1}{2} \rho \frac{\pi D^{2}}{4} V^{3}
$$

This is the total power available in the wind source.

On the other hand, the power extracted by the wind turbine is given by:

$$
P_{T}=T \omega
$$

The wind turbine power coefficient

$$
C_{p}=\frac{T \omega}{\frac{1}{2} \rho A V^{3}}=\lambda C_{m}
$$

where $\lambda$ is the tip speed ratio given by

$$
\lambda=\frac{\omega D}{2 V}
$$

And $C_{m}$ is the moment coefficient given by

$$
C_{m}=\frac{T}{\frac{1}{2} \rho A D V^{2}}
$$

Equation (13) will be used to analyze and validate the wind turbine efficiency.

According to Betz [10], the theoretical maximum power efficiency of any design of wind turbine is 0.59 . This is called Betz limit and wind turbines cannot operate at this maximum limit. The common real values of power coefficient for large scale wind turbines are $0.30-0.45$, however, small scale wind turbines are less efficient, and their power coefficients can be below this range.

When a shaft rotates, it may well go into transverse oscillations and the out of balance forces resulting in centrifugal force will induce the shaft to vibrate.

The centrifugal force is given by the following equation [10]

$$
F_{c}=M \omega^{2}(r+e)
$$

where $M$ is the mass, $r$ is the shaft deflection, $(r+e)$ is the distance from the center of gravity, and $\omega$ is the shaft angular velocity. 
The deflection force is given by

$$
F=K_{t} r
$$

where $K_{t}=M \omega_{n}^{2}$ is the transverse stiffness

Equating Equation (16) and Equation (17):

$$
K_{t} r=M \omega^{2}(r+e)
$$

After some algebraic manipulation, we get

$$
r=\frac{e}{\left(\frac{\omega_{n}}{\omega}\right)-1}
$$

where $\omega_{n}$ is the shaft natural frequency.

It is noticed that when the shaft rotates at an angular speed equal to the natural frequency of the transverse oscillations, its vibration becomes large and shows up as a whirling of the shaft.

Natural frequency is the number of times a system will oscillate (move back and forth) between its original position and its displaced position, if there is no outside interference. Wind turbine shaft can be regarded as simply supported where the ends are free to rotate normal to the axis, and the frequency of this kind of shaft is given by [16]:

$$
f=\frac{\pi}{2} n^{2} \sqrt{\frac{g E I}{W L}}
$$

where $n$ is the mode, $g$ is the constant of gravity, $E$ is the Young modulus, $I$ is the moment of inertia, $W$ is the shaft weight, and $L$ is the shaft length.

In the absence of damping, the dynamic character of the blade model can be expressed in matrix form as [16]:

$$
K V=\omega^{2} M V
$$

Here $K$ is the stiffness matrix, $M$ is the mass matrix, $\omega$ is the angular frequency of vibration for a given mode and $V$ is the mode vector that expresses the corresponding mode shape.

The resolution of this equation is not straight forward due to the complexity of the blade shape and incapability of finding the exact value of $K$.

\subsection{Finite Element Modal Analysis of HAWT Rotors and Shaft}

The imported pressure distributions for the HAWT rotors are presented in the Table 3. It can be noticed that the rotor's maxi-mum pressure distributions are located to the blade's tip as expected.

The HAWT rotors included the imported pressure as applied load, a remote displacement at the edges of the blade roots as support, and a rotational velocity of $50 \mathrm{rad} / \mathrm{sec}$. It is assumed that blades are connected to the HAWT hub by applying the remote displacement as support.

The imported moment from ANSYS Fluent to ANSYS structural environment 
Table 3. Imported pressure distributions.

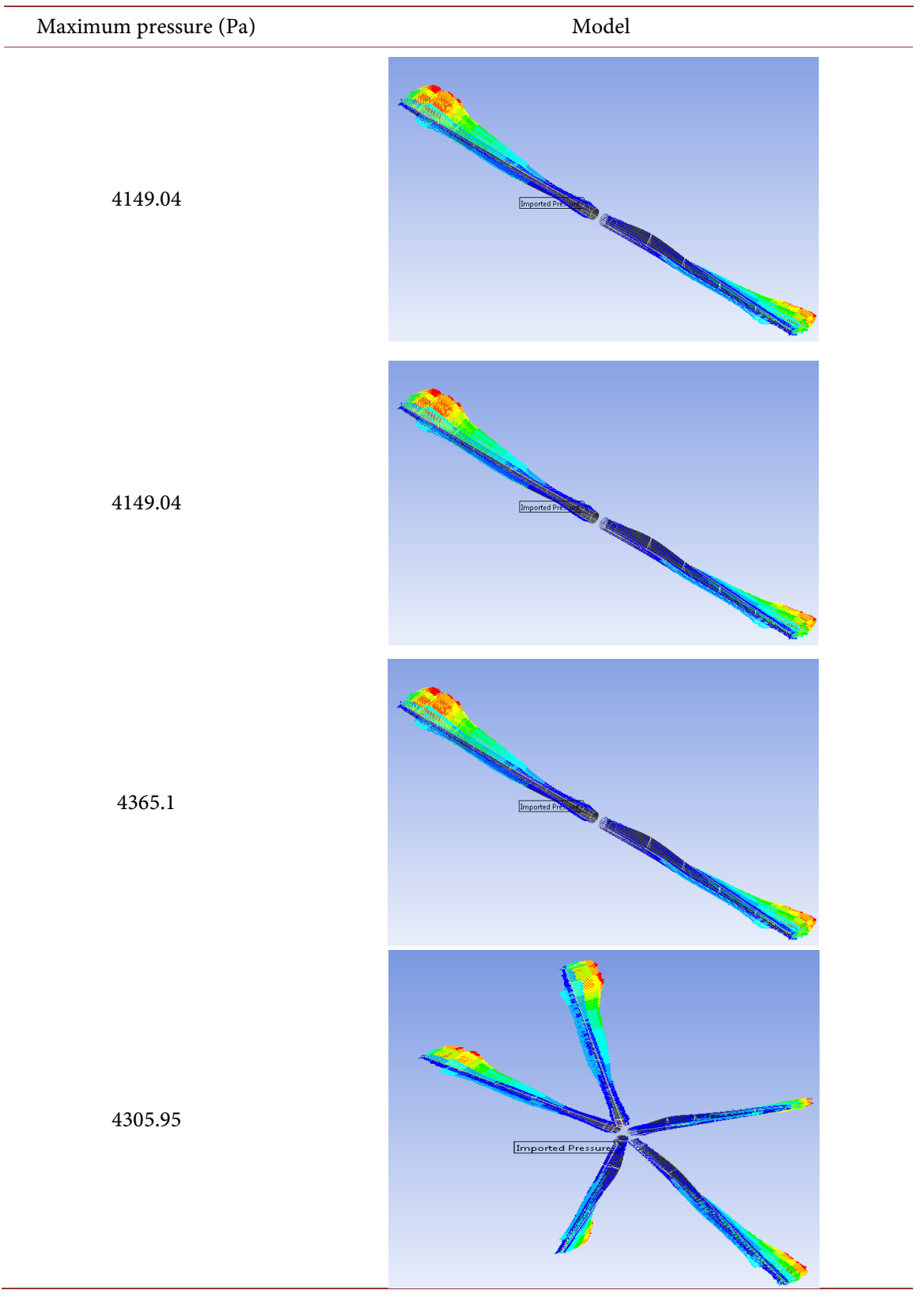

was monitored and used as an applied load during the shaft vibration investigation. The boundary conditions for the shaft included two fix supports applied at the top face of each bearing and a moment of $108.64 \mathrm{~N} \cdot \mathrm{m}$ corresponding to moment imported from ANSYS Fluent. The modal analysis of HAWT rotors and shaft has been performed using these boundary conditions.

\section{Results and Discussion}

\subsection{Aerodynamic Results of Entire HAWT}

The velocity and pressure contours around the entire HAWT are shown in Figure 11(a) and Figure 11(b) respectively. The maximum velocity and pressure 


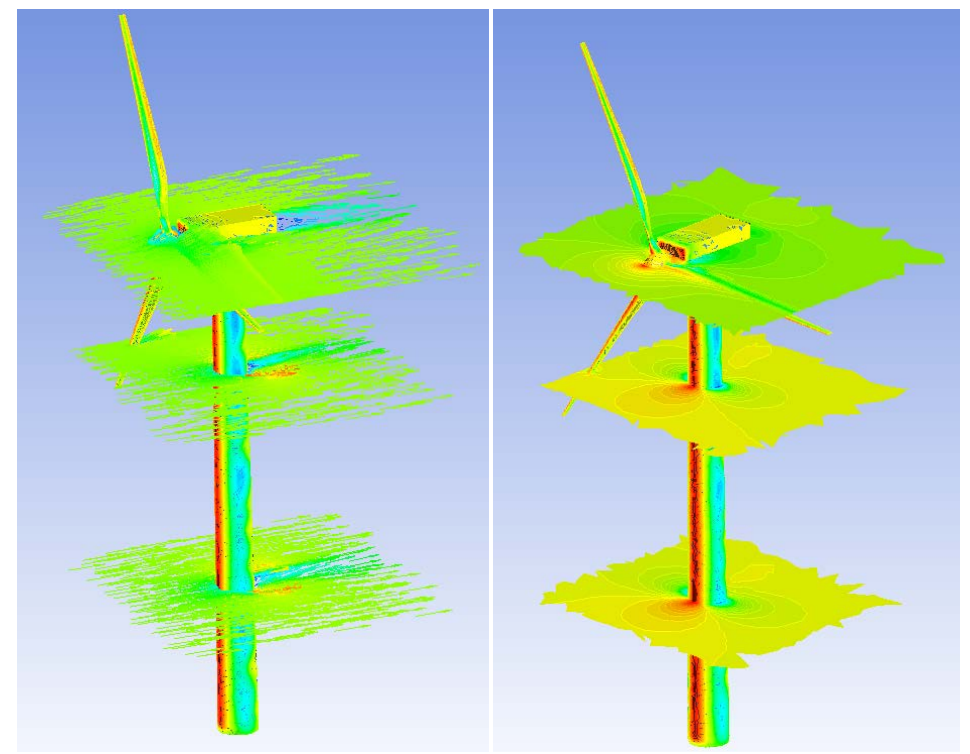

Figure 11. (a) Velocity contour around the entire HAWT surfaces. (b) Pressure contour around the entire HAWT surfaces.

contours are notice on the blades and tower front side.

\subsection{Aerodynamic Results of HAWT Rotors}

The pressure contours around the HAWT blade model are shown in Figure 12. The pressure contour near the blade root, blade middle, and blade tip are shown. The maximum pressure contours are located near the leading edge which is an indication of good blade design. The velocity contours near the blade root, blade middle, and blade tip are shown in Figure 13. The low flow turbulence is an indication of wind turbine high efficiency. The highest pressure difference is located near the middle of the blade model. The pressure distribution on the three HAWT blade models surface is presented in Figure 14. The maximum pressure distribution occurs near the leading edge of the blades which will produce high lift force and low drag force which is an indication of high power generation.

\subsection{Moment and Power Coefficients Results}

The moment coefficient variation with time of three different models at $4 \mathrm{~m} / \mathrm{s}, 6$ $\mathrm{m} / \mathrm{s}, 8 \mathrm{~m} / \mathrm{s}, 10 \mathrm{~m} / \mathrm{s}$, and $12 \mathrm{~m} / \mathrm{s}$ inlet wind velocities are shown in Figure 15. The power coefficient can be calculated by multiplying the average moment coefficient with the tip speed ratio. It can be seen from these figures that the three bladed HAWT rotor enjoys the highest average moment coefficient followed by five bladed HAWT rotor.

The moment coefficients of two, three, and five bladed HAWT rotors at five different inlet wind speeds have been compared and are shown in Figures 16-18 respectively. It can be noticed that the $8 \mathrm{~m} / \mathrm{s}$ inlet wind speed produced the highest average moment coefficient for all three models. This inlet wind speed can be considered as an optimum wind speed for the models. 


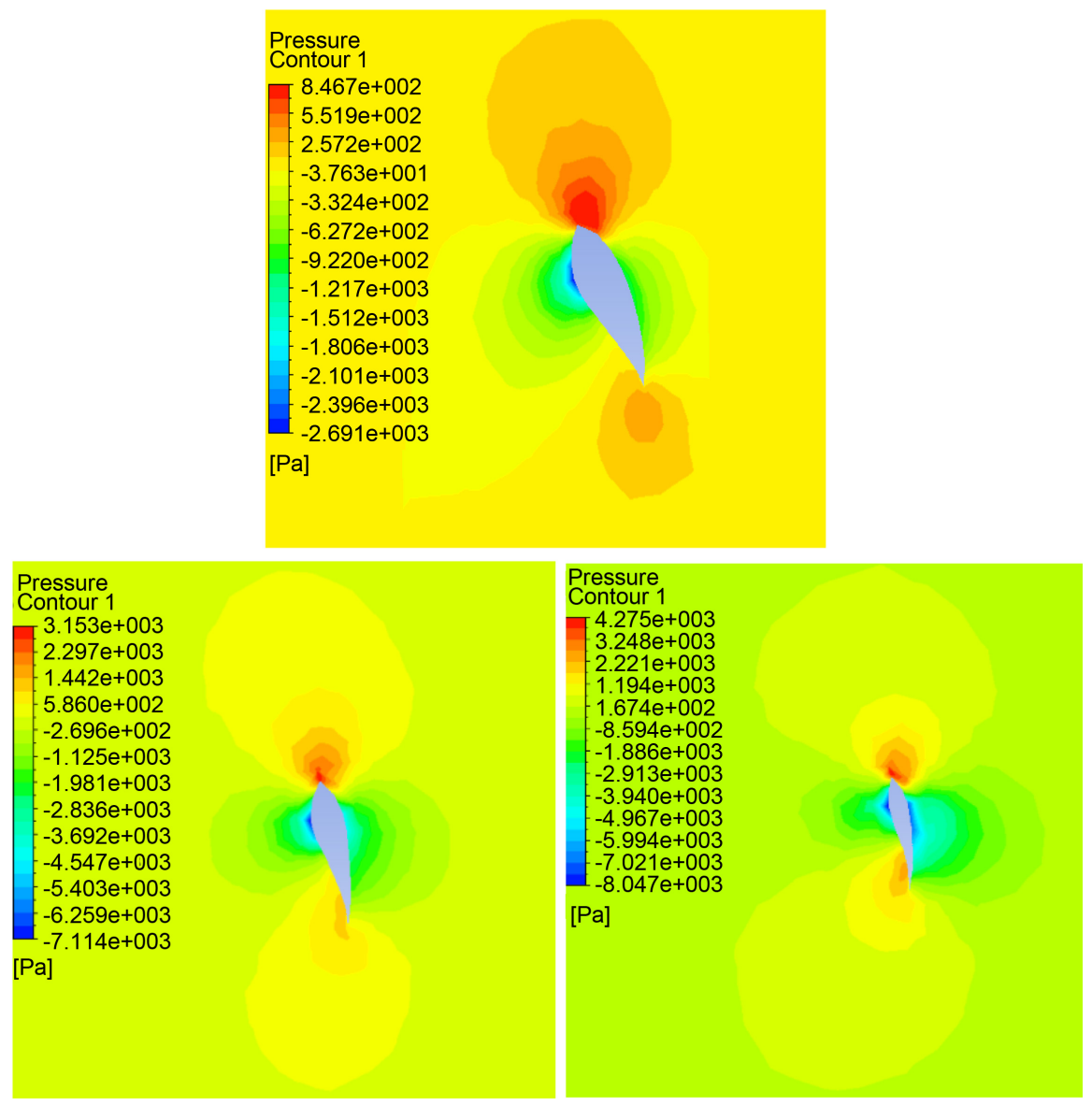

Figure 12. Pressure contour around the blade model surface.

Table 4 presents the power and the moment coefficients for three different HAWT models.

It is noticed that the highest moment and power coefficients values of 0.1527231 and 0.43192 respectively are attained by the three bladed HAWT rotor model at $8 \mathrm{~m} / \mathrm{s}$ inlet wind velocity. All power coefficients result for three blades HAWT are higher than those of two and five bladed HAWT rotors at the same tip speed ratio. The two-bladed HAWT rotor has the lowest moment and power coefficient of 0.034075711 and 0.1927 at $4 \mathrm{~m} / \mathrm{s}$ inlet velocity respectively. It is noticed that a large power coefficient increase with the increase of number of blades from two to three, whereas the power coefficient increase with the increase of blade number from three to five is relatively low. All power coefficient results for two blades HAWT are lower than those of three and five bladed HAWT rotors at the same tip speed ratio. The power coefficients as function of tip speed ratio for three HAWT rotor models have been plotted. The results are presented in Figure 19 where they are compared with those of Fei-Bin Hsiao et al. [10]. It is noticed that the three-bladed HAWT rotor attained the highest power coefficients of 0.43192 at 2.8281 tip speed ratio (TSR). It can also be noticed that a high increase of power coefficient with the increase of number of blades from two to three, but the increase of power coefficient with the increase 


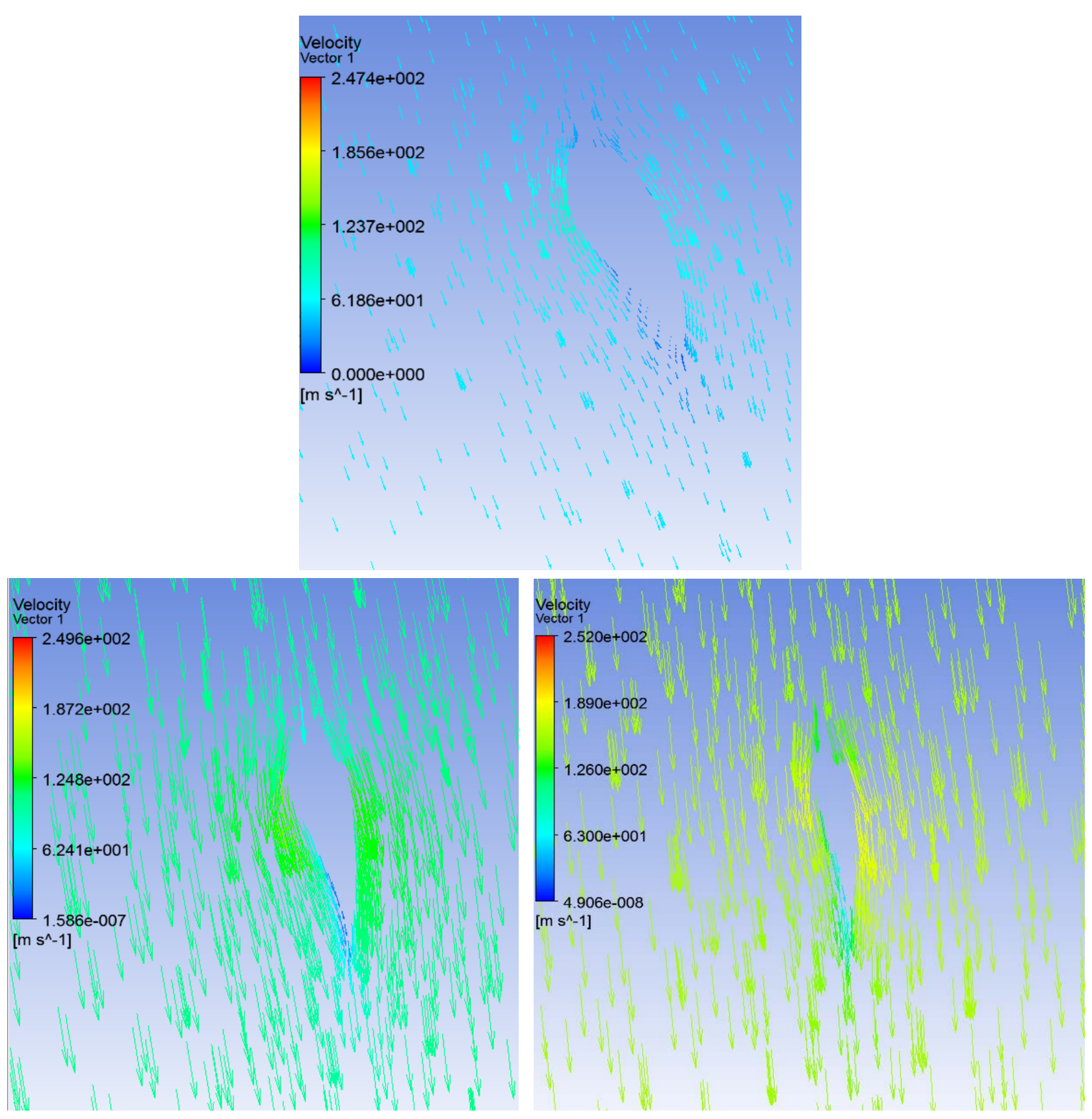

Figure 13. Velocity contour around the blade model surface.

of blades from three to five is relatively low as mentioned earlier. Fei-Bin Hsiao et al. [10] highest power coefficient of 0.428 was attained at 4.92 tip speed ratio. This comparison shows that the current HAWT design has the higher power coefficient than the previous results.

\subsection{HAWT Modal Analysis Results}

The entire HAWT and shaft deflection simulations results are shown in Figure 20. The maximum deflection occurred on the HAWT blade and in the shaft center. 

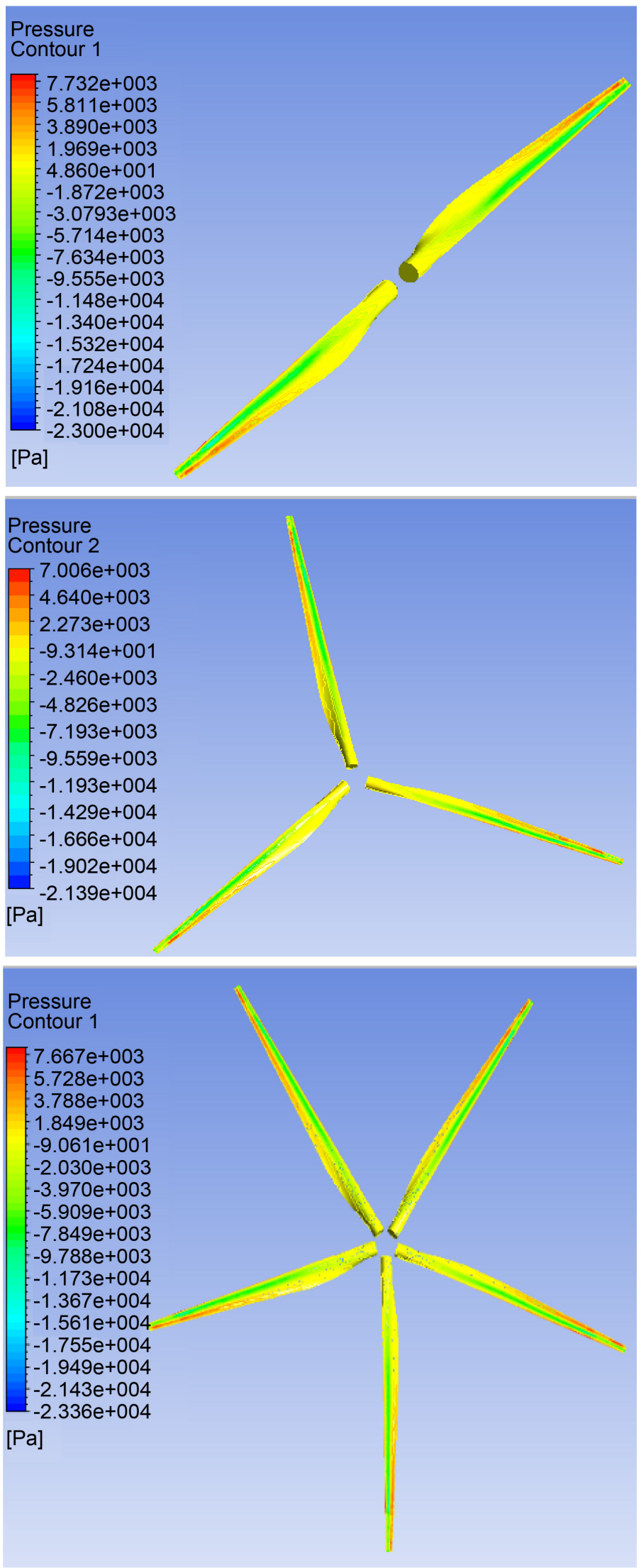

Figure 14. Pressure distribution on the HAWT blade models surface. 


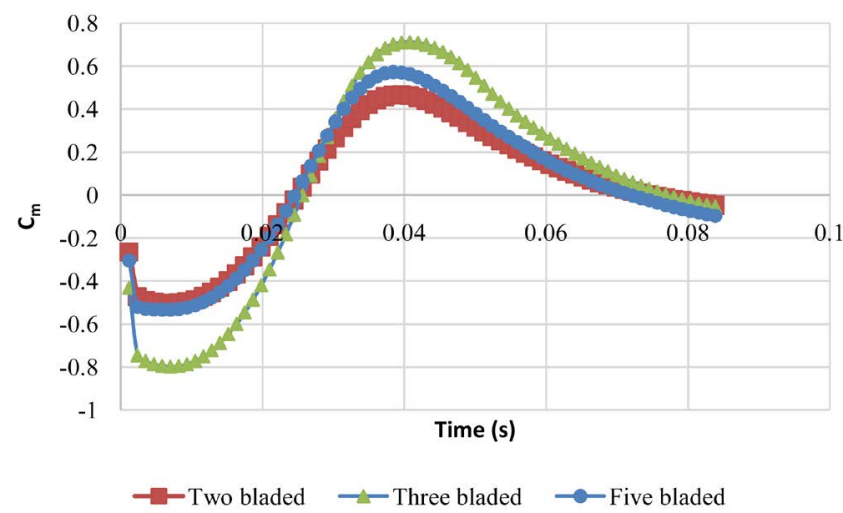

(a)

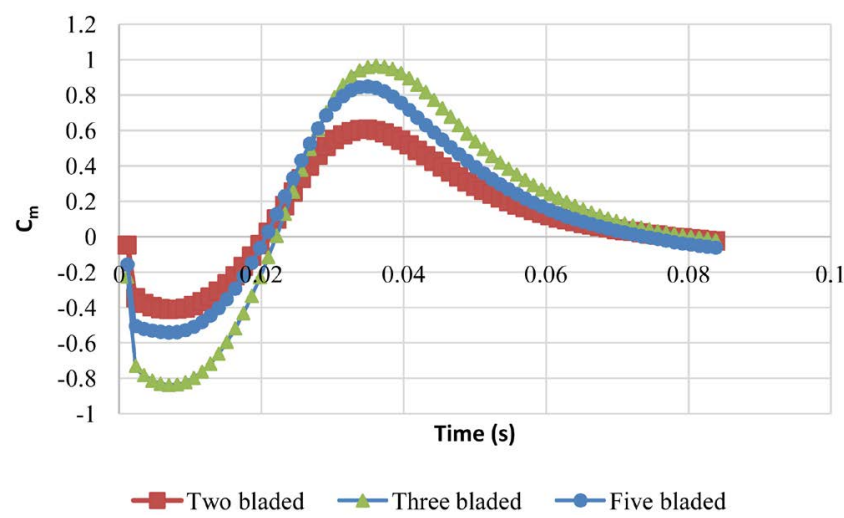

(c)

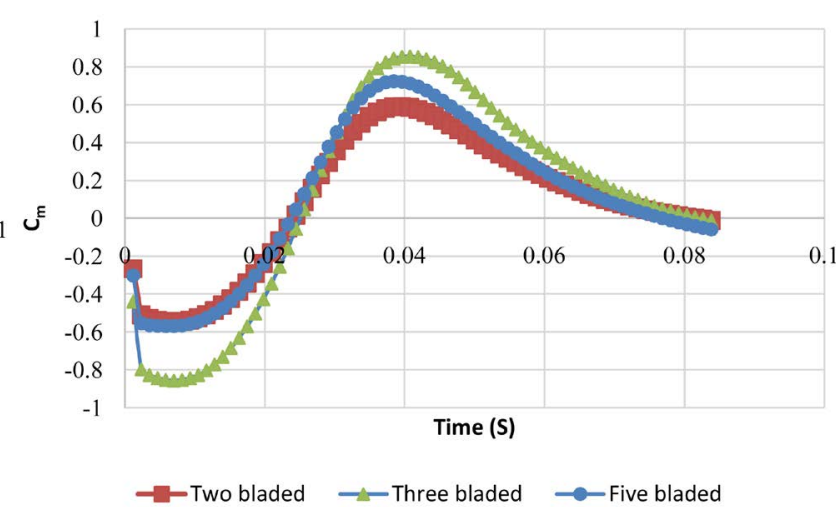

(b)

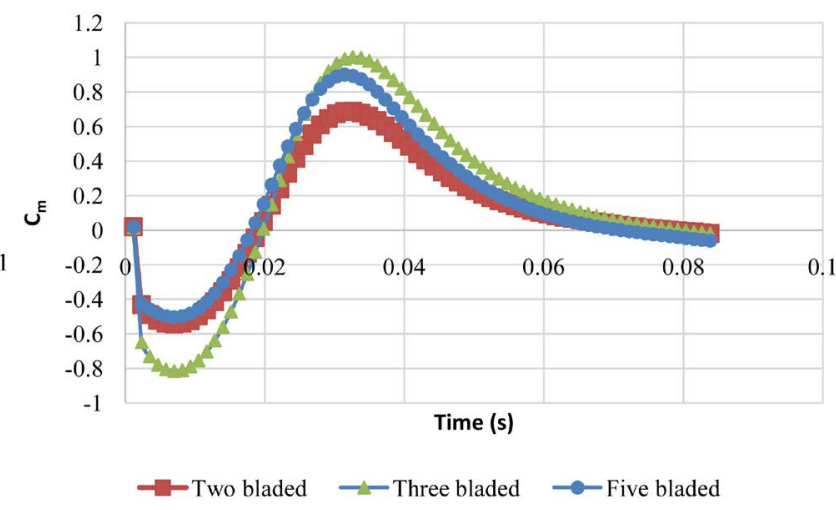

(d)

Moment coefficients for $12 \mathrm{~m} / \mathrm{s}$ inlet velocity

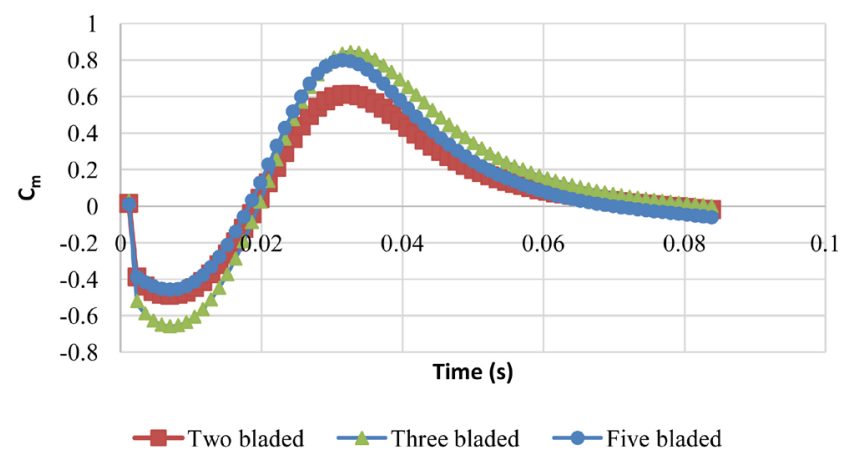

(e)

Figure 15. Variation of Moment coefficient with time at $4 \mathrm{~m} / \mathrm{s}, 6 \mathrm{~m} / \mathrm{s}, 8 \mathrm{~m} / \mathrm{s}, 10 \mathrm{~m} / \mathrm{s}$ and $12 \mathrm{~m} / \mathrm{s}$ inlet wind velocities. (a) For 4 $\mathrm{m} / \mathrm{s}$, (b) For $6 \mathrm{~m} / \mathrm{s}$. (c) For $8 \mathrm{~m} / \mathrm{s}$. (d) For $10 \mathrm{~m} / \mathrm{s}$. (e) For $12 \mathrm{~m} / \mathrm{s}$.

The two, three, and five HAWT rotors deflection simulations results are shown in Figures 21-23 respectively. The maximum deflection was notice on the blade tip while the minimum was notice near the root. Also, Table 5 shows the HAWT and shaft natural frequency at different mode shapes.

Table 6 shows the two, three, and five bladed rotors natural frequency at different mode shapes. Aluminum alloy and homogenized composite material used in real wind turbine blade were used.

The density and orthopedic properties of GE 1.5 XLE wind turbine were used 


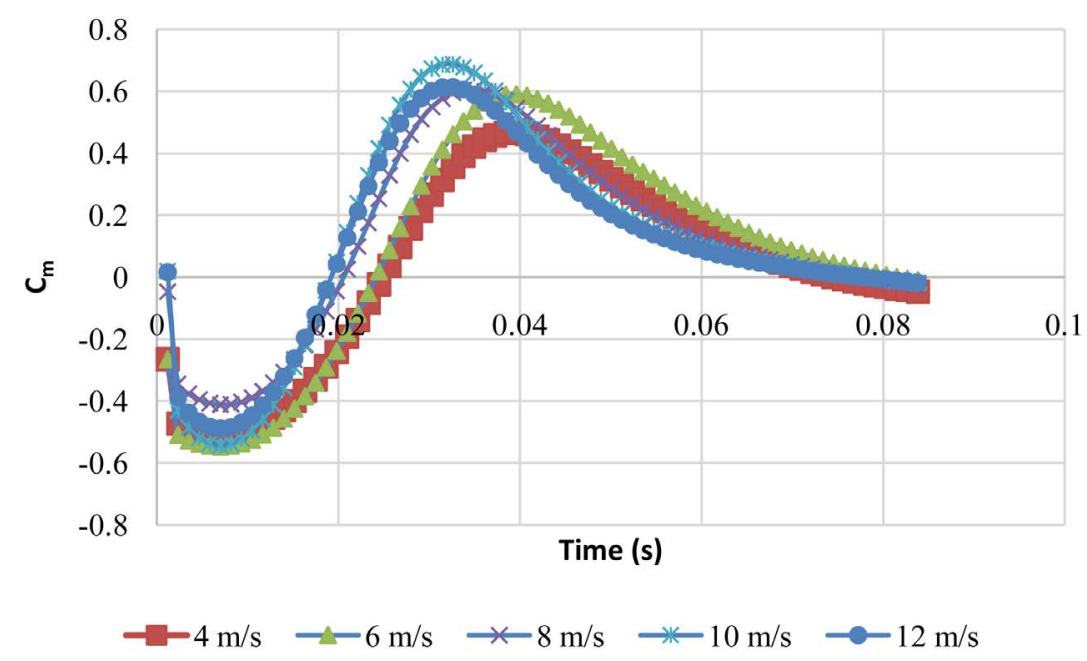

Figure 16. Moment coefficients variation of two bladed HAWT rotor.

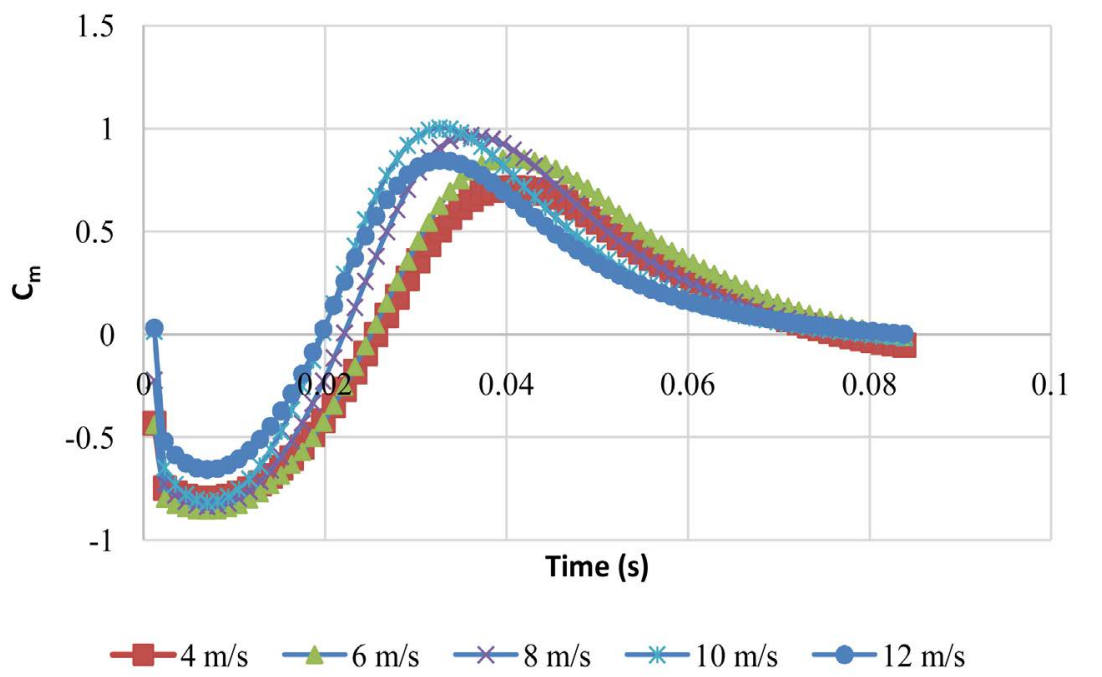

Figure 17. Moment coefficient for three-bladed HAWT model.

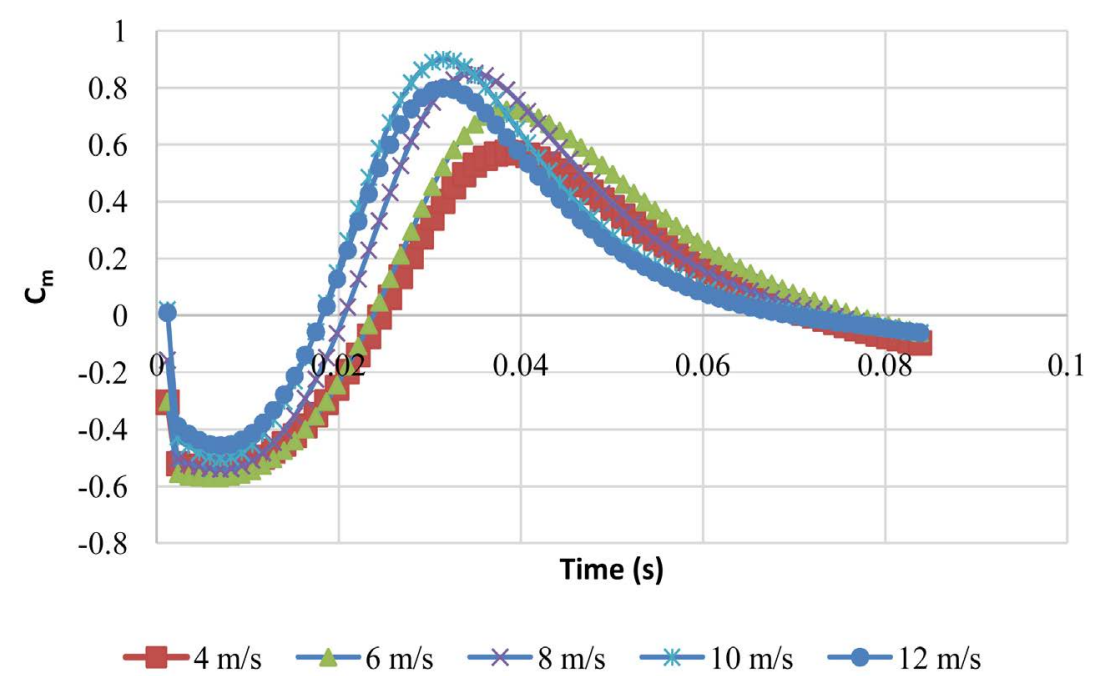

Figure 18. Moment coefficient for five-bladed HAWT model. 

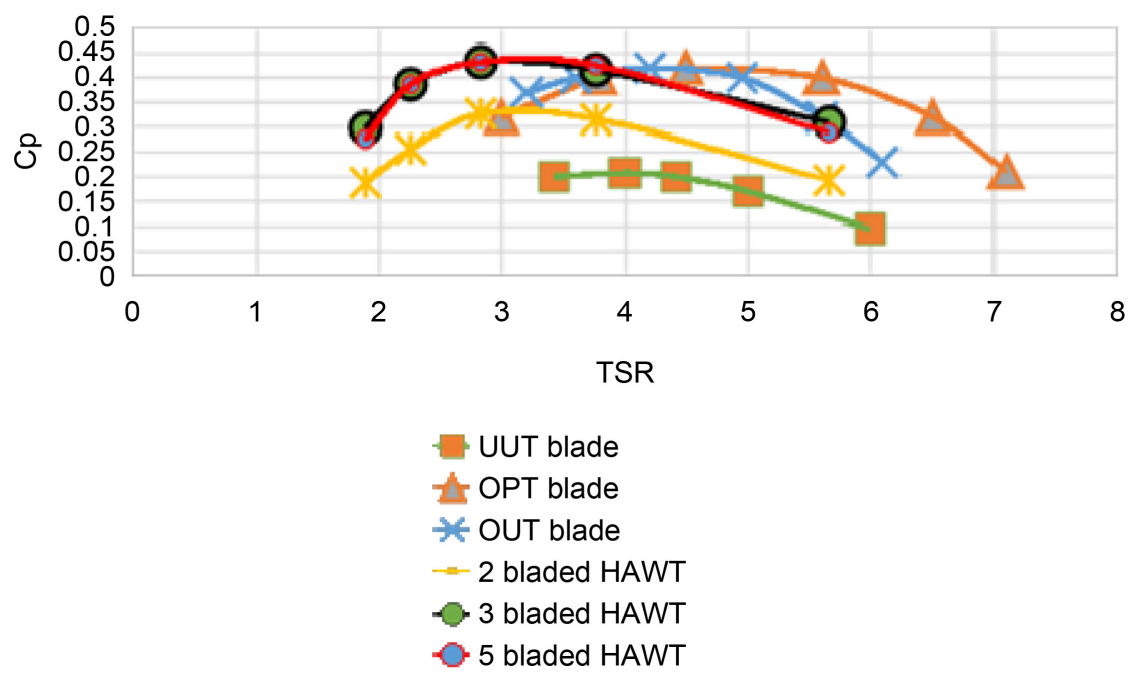

Figure 19. Fei-Bin Hsiao et al. (2013) and current power coefficients comparison.
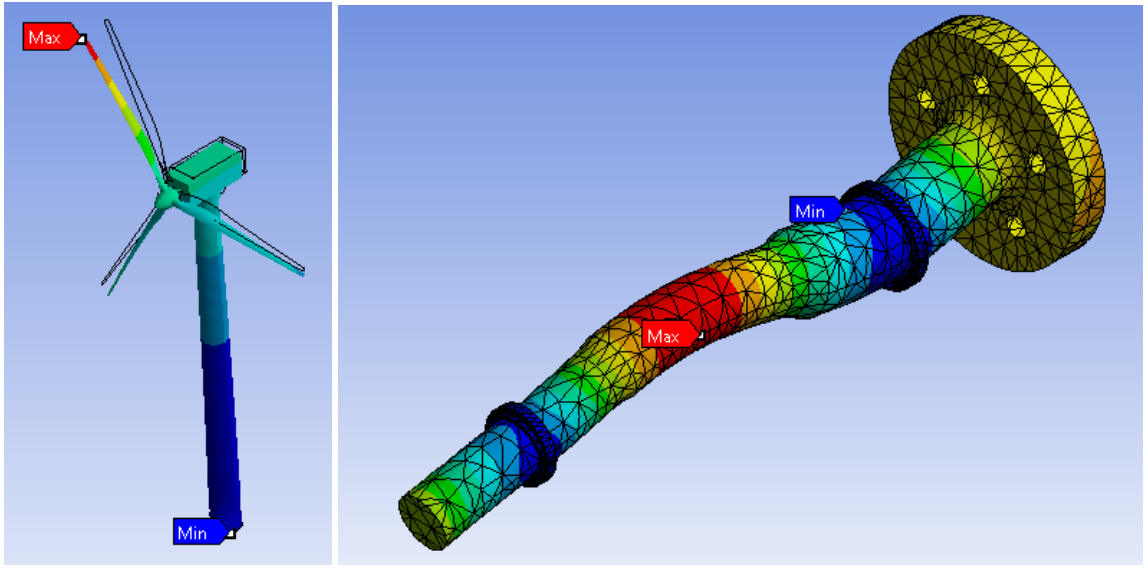

Figure 20. Entire HAWT and shaft deflection.

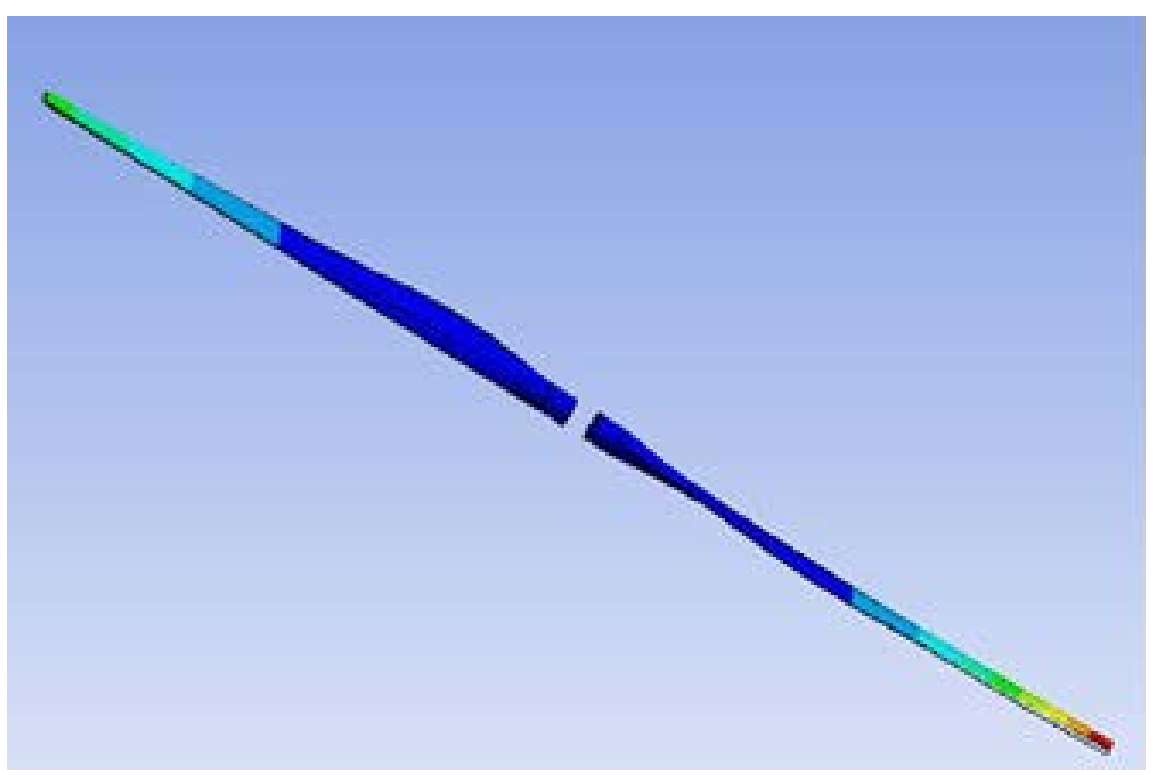

Figure 21. Two-bladed HAWT rotor deflection. 


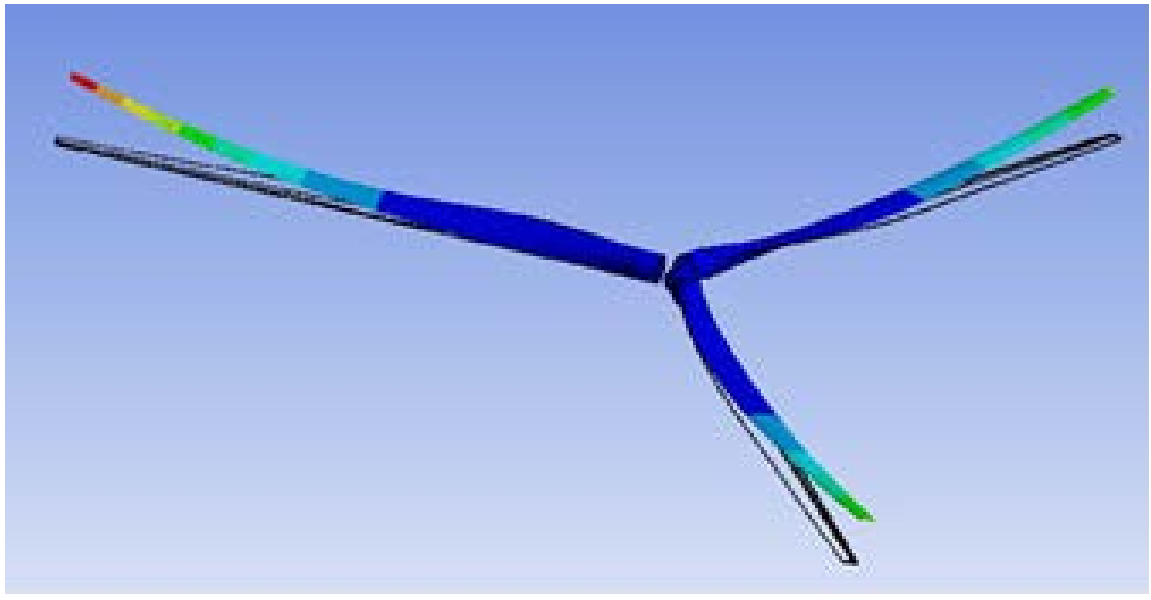

Figure 22. Three-bladed HAWT rotor deflection.

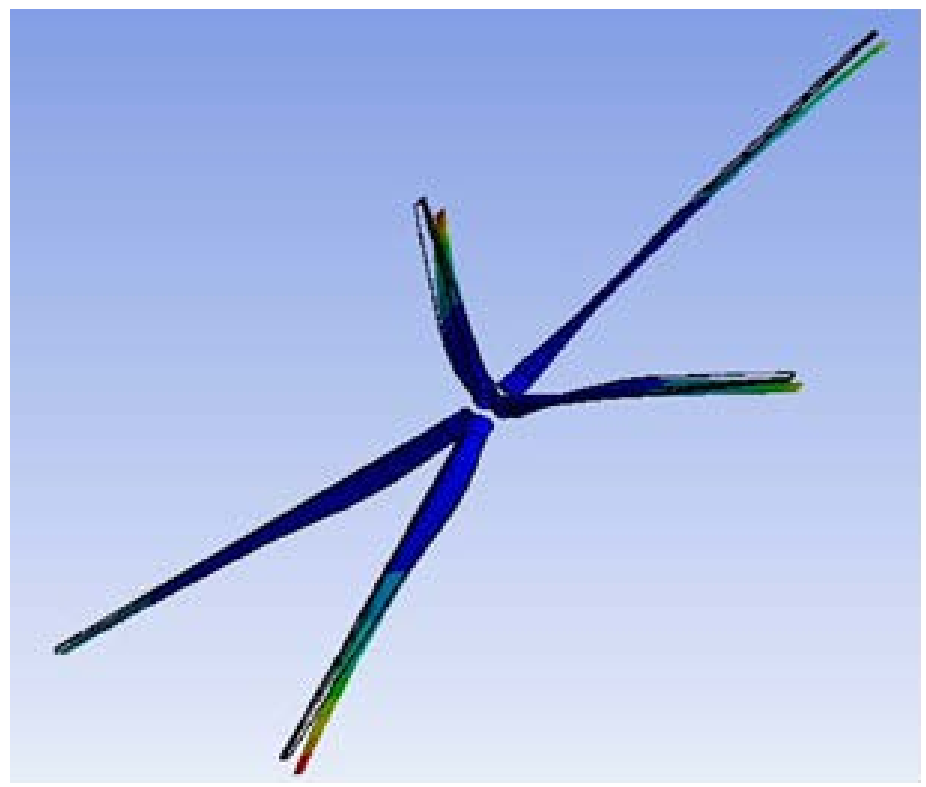

Figure 23. Five-bladed HAWT rotor deflection.

in this research. An increase of natural frequency is observed if a composite material is used instead of aluminum alloy material.

Detail figures on natural frequencies and mode shapes of HAWT, Shaft and blades are shown in Appendix A.

The computational domain consists of a rotating zone surrounding the blades and a stationary far-field zone. A mesh interface is created between the two zones. The interface is necessary because the nodes on the boundaries of the far-field and rotational zones are intentionally non-conformal. The interface pairs these so that interpolation can occur, and fluid may pass into and out of the rotating region. For each case, a static simulation with moving reference frame (MRF) and a dynamic sliding mesh model (SMM) are completed. The rotation is first defined using the steady-state solver with MRF, and the simulation is then solved in a transient manner using a sliding mesh motion. The converged 
Table 4. Power and moment coefficients for three different HAWT models.

\begin{tabular}{ccccc}
\hline Model & $\mathrm{V}(\mathrm{m} / \mathrm{s})$ & TSR & Cm (Average) & Cp (Average) \\
\hline \multirow{2}{*}{2 bladed HAWT } & 12 & 1.8854 & 0.099712707 & 0.188 \\
& 10 & 2.2625 & 0.113038493 & 0.25575 \\
& 8 & 2.8281 & 0.115454155 & 0.3265 \\
3 bladed HAWT & 6 & 3.7708 & 0.083750718 & 0.3158 \\
& 4 & 5.65625 & 0.034075711 & 0.1927 \\
\hline & 12 & 1.8854 & 0.158055249 & 0.298 \\
& 10 & 2.2625 & 0.170921547 & 0.38671 \\
\hline 5 bladed HAWT & 6 & 2.8281 & 0.1527231 & 0.43192 \\
& 12 & 3.7708 & 0.1103019 & 0.41593 \\
& 10 & 5.65625 & 0.054628062 & 0.30899 \\
\hline & 6 & 1.8854 & 0.146185194 & 0.27562 \\
& 6 & 2.2625 & 0.170921547 & 0.38671 \\
\hline
\end{tabular}

Table 5. HAWT and shaft natural frequency at different mode shapes.

\begin{tabular}{cccccc}
\hline & \multicolumn{3}{c}{ Computed Natural frequency } & \multicolumn{2}{c}{ Calculated natural frequency } \\
\hline Mode & HAWT & Shaft $^{1}$ & Shaft $^{2}$ & Shaft $^{1}$ & Shaft $^{2}$ \\
\hline 1 & 0.39971 & 1338.30 & 1346.95 & 1000.827431 & 1003.848837 \\
2 & 0.48004 & 3970.55 & 3782.20 & 4003.309722 & 4015.395349 \\
3 & 0.48283 & 8985.30 & 8998.85 & 9007.446875 & 9034.639535 \\
4 & 0.60208 & $16,149.80$ & $16,322.90$ & $16,013.23889$ & $16,061.5814$ \\
5 & 0.61122 & $24,256.80$ & $24,429.55$ & $25,020.68576$ & $25,096.22093$ \\
6 & 0.85593 & $35,583.65$ & $35,760.50$ & $36,029.7875$ & $36,138.55814$ \\
\hline
\end{tabular}

Table 6. HAWT rotors natural frequency at different mode shapes.

\begin{tabular}{|c|c|c|c|c|c|c|}
\hline \multirow[b]{2}{*}{ Mode } & \multicolumn{5}{|c|}{ Natural frequency } & \multirow[b]{2}{*}{$\begin{array}{c}\text { Five bladed } \\
\text { rotor }^{2}\end{array}$} \\
\hline & $\begin{array}{l}\text { Two } \\
\text { bladed } \\
\text { rotor }^{1}\end{array}$ & $\begin{array}{c}\text { Two bladed } \\
\text { rotor }^{2}\end{array}$ & $\begin{array}{l}\text { Three } \\
\text { bladed } \\
\text { rotor }^{1}\end{array}$ & $\begin{array}{c}\text { Three } \\
\text { bladed } \\
\text { rotor }^{2}\end{array}$ & $\begin{array}{c}\text { Five bladed } \\
\text { rotor }^{1}\end{array}$ & \\
\hline 1 & 49.336 & 74.269 & 14.5525 & 19.457 & 9.2613 & 12.407 \\
\hline 2 & 50.158 & 76.544 & 14.5647 & 19.133 & 9.2614 & 12.598 \\
\hline 3 & 69.737 & 97.697 & 16.574 & 21.866 & 9.6173 & 13.8 \\
\hline 4 & 104.59 & 129.998 & 17.947 & 23.074 & 9.6449 & 13.986 \\
\hline 5 & 106.99 & 157.775 & 40.983 & 52.588 & 22.396 & 34.763 \\
\hline 6 & 137.24 & 185.936 & 42.154 & 55.986 & 25.408 & 35.757 \\
\hline
\end{tabular}


static result from the MRF simulation is used to initialize the transient SMM solver. Example graphs of the residuals for MRF and SMM simulations can be seen in Figure 24. Convergence criteria are kept consistent throughout the study requiring all 5 residuals to decrease to a value of $1 \mathrm{e}-03$.

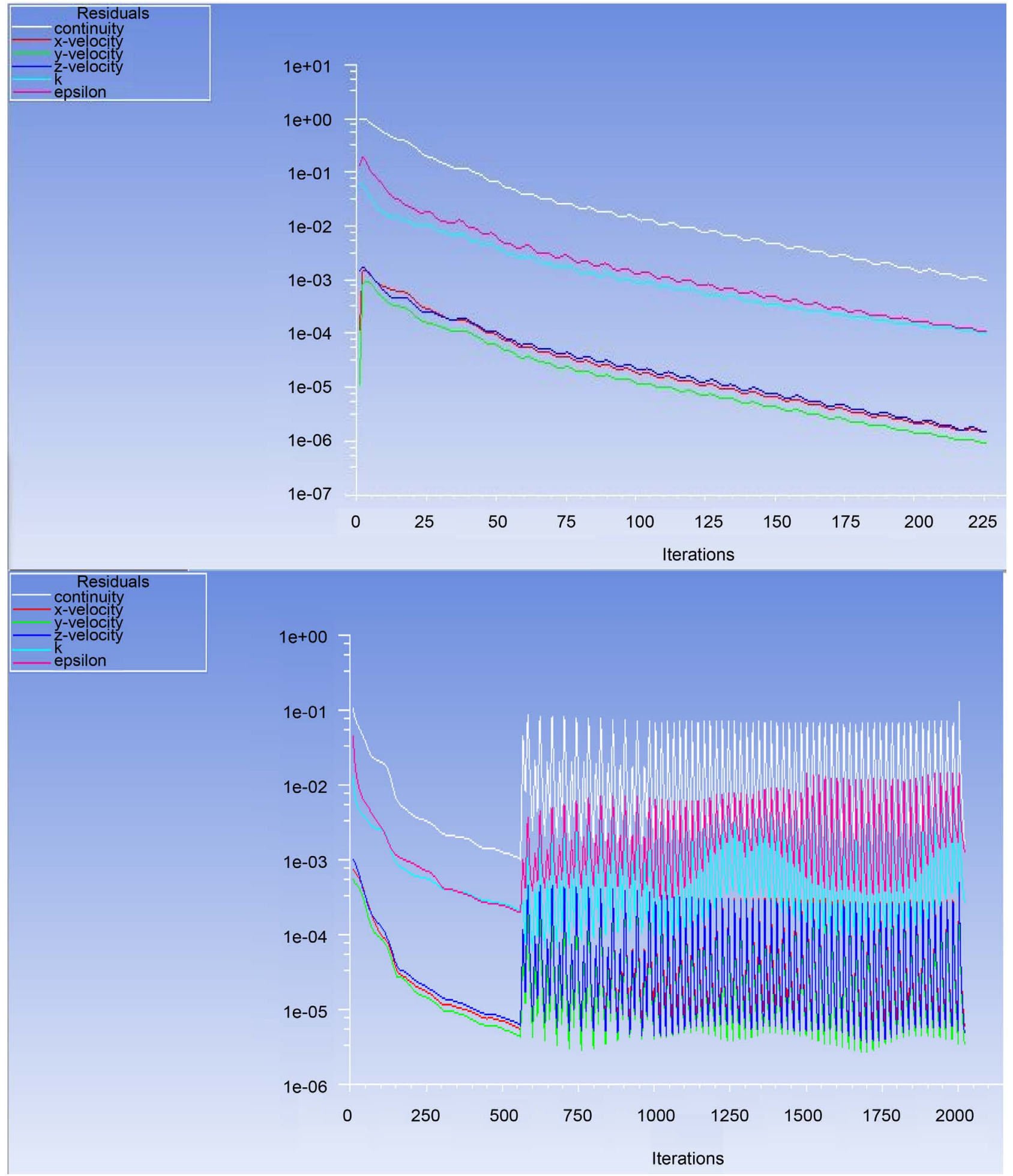

Figure 24. MRF residuals converged after 225 iterations and SMM residuals. 
For the transient solver, coefficients of moment $(\mathrm{Cm})$ are monitored over time with accurate reference values. Time step size is dependent on the RPM value for each case. Time steps are calculated to account for every 10 degrees of model rotation. For 2 full rotations, 72 time steps per simulation are run with 20 iterations per time step.

Boundary conditions for the simulations are taken from experimental data. These include air velocity inlet speed and corresponding rotational speed of the blades. The pressure outlet is kept at constant atmospheric pressure. The blade walls are given a no slip condition and zero rotational velocity relative to the sliding mesh zone (equal to the rotating fluid domain).

The realizable k-epsilon model is used with the SIMPLE segregated algorithm. The SIMPLE algorithm uses a relationship between velocity and pressure corrections to enforce mass conservation and to obtain the pressure field (ANSYS Fluent Theory Guide, 2012). For improved accuracy, the double precision option is selected as well as second order upwind based discretization for mean flow, turbulence, and transition equations.

\section{Conclusions}

The efficiency of small scale HAWT with various numbers of blades has been investigated in this study and this efficiency is explained by the fact that putting more than three blades on the HAWT rotor will be the waste of material and money while reducing the HAWT performance. An increase of HAWT performance was observed if three bladed HAWT was used instead of two bladed HAWT and the change of performance was relatively low or the same for the five bladed HAWT.

The HAWT vibration is a big challenge on the HAWT performance and the choice of material to use is crucial during the de-sign process. Homogenized composite material has proven the highest performance due to its resistance to wind load. An average natural frequency increase of $41.7 \%$, 31\%, and $42.4 \%$ was observed for two, three, and five HAWT rotors if the homogenized composite material is used in wind turbine blade instead of aluminum alloy. Homogenization of material can be suggested during the blade design to increase its stiffness and contribute in its wind air resistance and performance.

Aluminum alloy has proven better performance than structural steel material on the HAWT shaft vibration; however, the difference in natural frequency is relatively low. The calculated average natural frequency of $0.3 \%$ was observed while the computed value of $0.2 \%$ was attained if aluminum alloy material is used instead of structural steel. This can be explained by the highest aluminum young modulus to density ratio compare to that of structural steel material. This quantity was used during the calculation of natural frequencies at different mode shapes. In the paper, the blade was created by modifying the large-scale GE 1.5XLE turbine to enhance the performance of the turbine which is applicable for the real size. But, we made it by this way to suit our laboratory capabilities 
and equipment. The conclusion obtained in this paper also useful for large scale HAWTs as well. The size of the computational domains is large enough to get these accurate results. The model is not created in the real size to fit our wind tunnel for testing purposes. This work is useful for large scale HAWTs too but within scale. Moreover, this is ingoing work for detailed vibration analysis with the detailed parameters of the blade (chords and twists distributions) and material properties of different parts of the turbine to be published in the next paper.

\section{Acknowledgements}

Authors would express the heartiest deep sense of gratitude to Mechanical Engineering Department of Georgia Southern University. Authors acknowledge NSF-RET: ENgaging Educators in Renewable EnerGY (ENERGY), Award \#1609524.

\section{References}

[1] (2008) 20\% Wind Energy by 2030. [Electronic Resource]: Increasing Wind Energy's Contribution to U.S. Electricity Supply. n.p.: U.S. Dept. of Energy, Energy Efficiency and Renewable Energy, Washington DC.

[2] Tasri, A. and Susilawati, A. (2014) Original Research Article: Selection among Renewable Energy Alternatives Based on a Fuzzy Analytic Hierarchy Process in Indonesia. Sustainable Energy Technologies and Assessments, 7, 34-44. https://doi.org/10.1016/j.seta.2014.02.008

[3] Hordon, R.M. (2015) Atmospheric Sciences. Salem Press Encyclopedia of Science Research Starters.

[4] Mohamad, A., et al (2014) Review of Analysis on Vertical and Horizontal Axis Wind Turbines. Applied Mechanics \& Materials, 695, 801-805. https://doi.org/10.4028/www.scientific.net/AMM.695.801

[5] Jeong, M.-S., Cha, M.-C., Kim, S.-W. and Lee, I. (2015) Numerical Investigation of Optimal Yaw Misalignment and Collective Pitch Angle for Load Imbalance Reduction of Rigid and Flexible HAWT Blades under Sheared Inflow. Energy, 84, 518-532. https://doi.org/10.1016/j.energy.2015.03.016

[6] Leithead, W. and Connor, B. (2010) Control of Variable Speed Wind Turbines: Dynamic Models. International Journal of Control, 73, 1173-1189. https://doi.org/10.1080/002071700417830

[7] Murtagh, P.J., Ghosh, A., Basu, B. and Broderick, B.M. (2008) Passive Control of Wind Turbine Vibrations Including Blade/Tower Interaction and Rotationally Sampled Turbulence. Wind Energy, 11, 305-317. https://doi.org/10.1002/we.249

[8] Kumara, A., Dwivedia, A., Paliwala, V. and Patil, P.P. (2014) Free Vibration Analysis of $\mathrm{Al} 2024$ Wind Turbine Blade Designed for Uttarakhand Region Based on FEA. Procedia Technology, 14, 336-347. https://doi.org/10.1016/j.protcy.2014.08.044

[9] Duque, E.P.N. and Michael, D.B. (2003) Navier-Stokes and Comprehensive Analysis Performance Predictions of the NREL Phase VI Experiment. Journal of Solar Energy Engineering, 125, 457-467. https://doi.org/10.1115/1.1624088

[10] Hsiao, F.-B., Bai, C.-J. and Chong, W.-T. (2013) The Performance Test of Three Different Horizontal Axis Wind Turbine (HAWT) Blade Shapes Using Experimental and Numerical Methods. Energies, 6, 2784-2803. 
https://doi.org/10.3390/en6062784

[11] Li, D.S., Li, R.N., Wei, L.J., Wang, X.Y., Qiang, Y. and Li, Y.R. (2013) Comparison of the Pressure Distribution of a Wind Turbine Blade Based on Field Experiment and CFD. IOP Conf. Series: Materials Science and Engineering 52 vol. 052004.

[12] Sagol, E., Reggio, M. and Ilinca, A. (1989) Determination of Elastic Twist in Horizontal Axis Wind Turbines (HAWTs). ISRN Mechanical Engineering, 2012, Article ID: 428671.

[13] Bai, C.J., Hsiao, F.B., Li, M.H., Huang, G.Y. and Chen, Y.J. (2013) Design of 10 kW Horizontal-Axis Wind Turbine (HAWT) Blade and Aerodynamic Investigation Using Numerical Simulation. Procedia Engineering, 67, 279-287.

https://doi.org/10.1016/j.proeng.2013.12.027

[14] Shah, T., Prasad, R. and Damodaran, M. (2013) Computational Modeling of Wind Energy Systems. Proceedings of the 8 th Asia-Pacific Conference on Wind Engineering ( $A P C W E$-VIII), Chennai, 10-14 December 2013. https://doi.org/10.3850/978-981-07-8012-8_255

[15] Rahman, M., Johnson, J., Pate, D., Sawinski, J., Seeloff, T., Ball, J., Molina, G., El Shahat, A. and Soloiu, V. (2016) Finite Element Structural Analysis of Commonly Used Horizontal Axis Wind Turbine Airfoils of Various Geometries. ASME 2016 International Mechanical Engineering Congress and Exposition (IMECE), Phoenix Convention Center, Phoenix, AZ, 11-17 November 2016, V010T13A017. https://doi.org/10.1115/IMECE2016-66958

[16] Wang, T.G., Wang, L., Zhong, W., Xu, B.F. and Chen, L. (2012) Large-Scale Wind Turbine Blade Design and Aerodynamic Analysis. Chinese Science Bulletin Kexue Tongbao No. 5.

\section{Nomenclature}

$\begin{array}{ll}\text { Symbol } & \text { Explanation } \\ A & \text { Rotor Area }\left(\mathrm{m}^{2}\right) \\ D & \text { Rotor diameter }(\mathrm{m}) \\ V & \text { Wind velocity }(\mathrm{m} / \mathrm{s}) \\ \rho & \text { Air density }\left(\mathrm{kg} / \mathrm{m}^{3}\right) \\ \omega & \text { Angular velocity }(\mathrm{rad} / \mathrm{sec}) \\ R e & \text { Reynolds Number } \\ T & \text { Torque }(\mathrm{Nm}) \\ P & \text { Power }(\mathrm{W}) \\ C_{p} & \text { Power coefficient } \\ N & \text { Revolutions per minute } \\ \operatorname{TSR}(\lambda) & \text { Tip speed ratio } \\ C_{m} & \text { Moment coefficient }\end{array}$




\section{Natural Frequencies and Mode Shapes}

\section{A1. HAWT and Shaft Natural Frequencies and Mode Shapes}

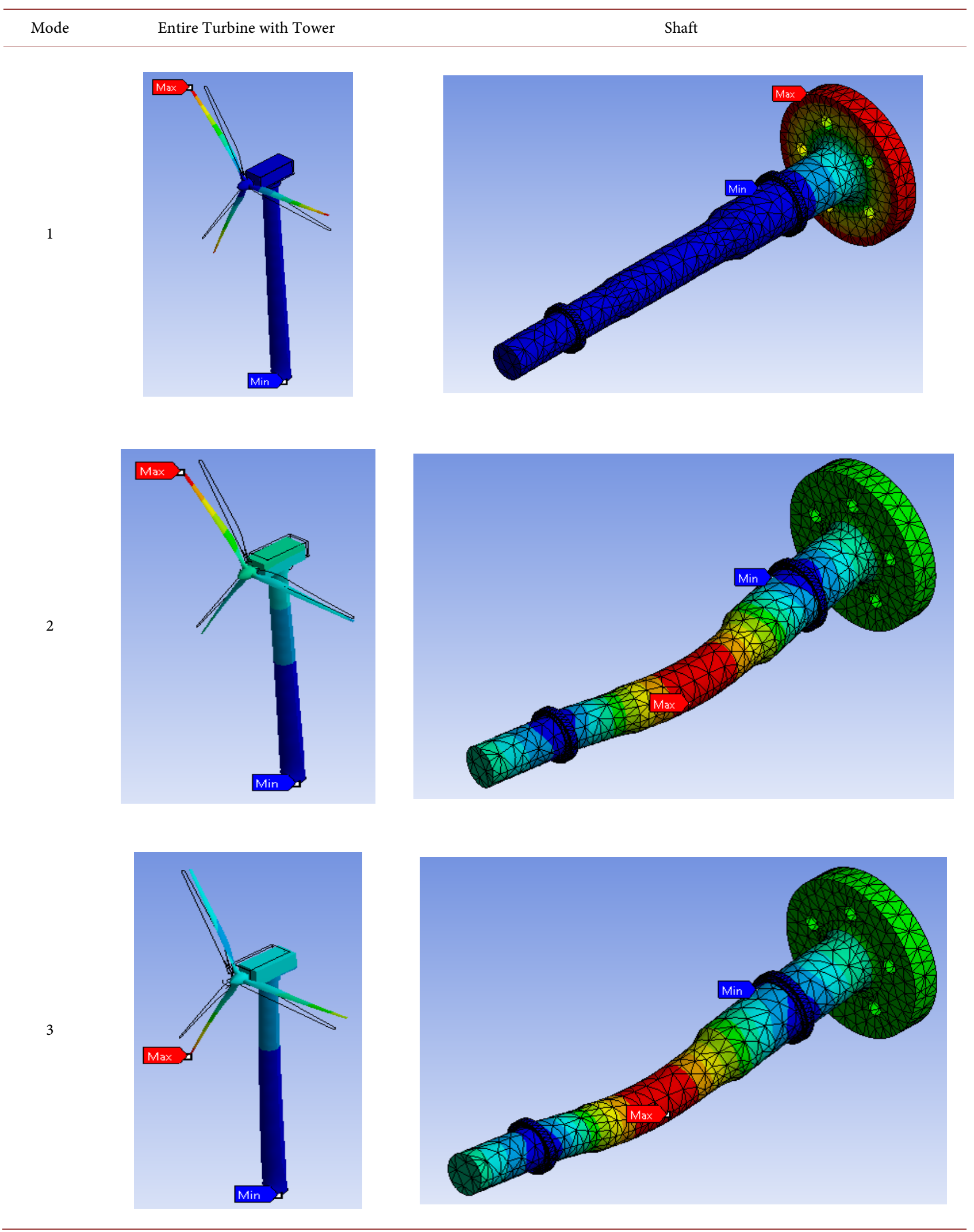




\section{Continued}

4
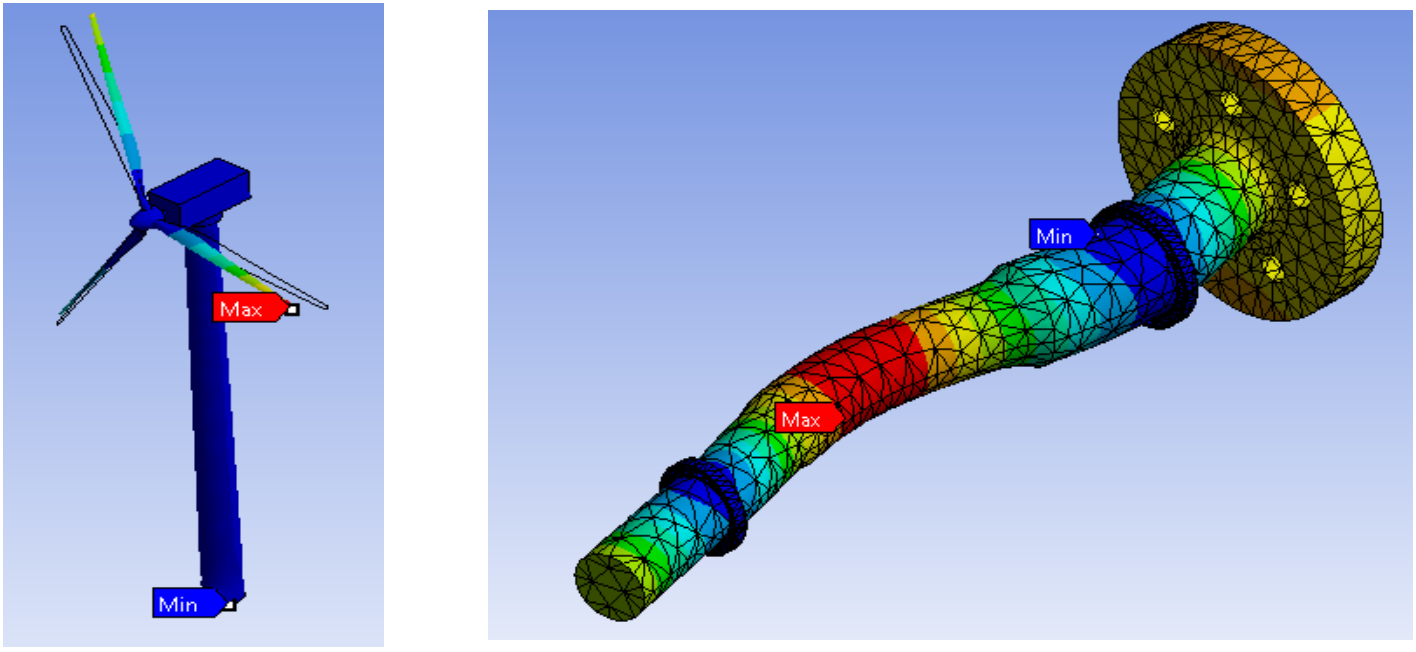

5
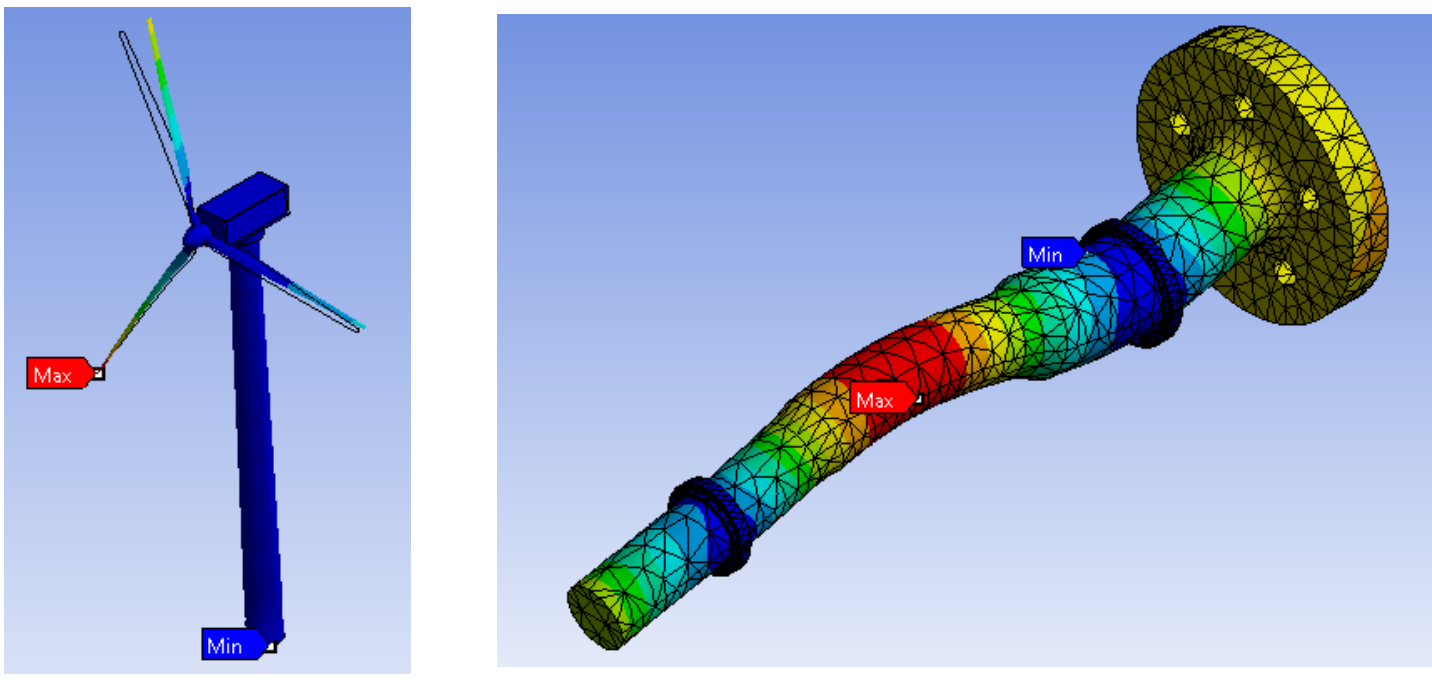

6
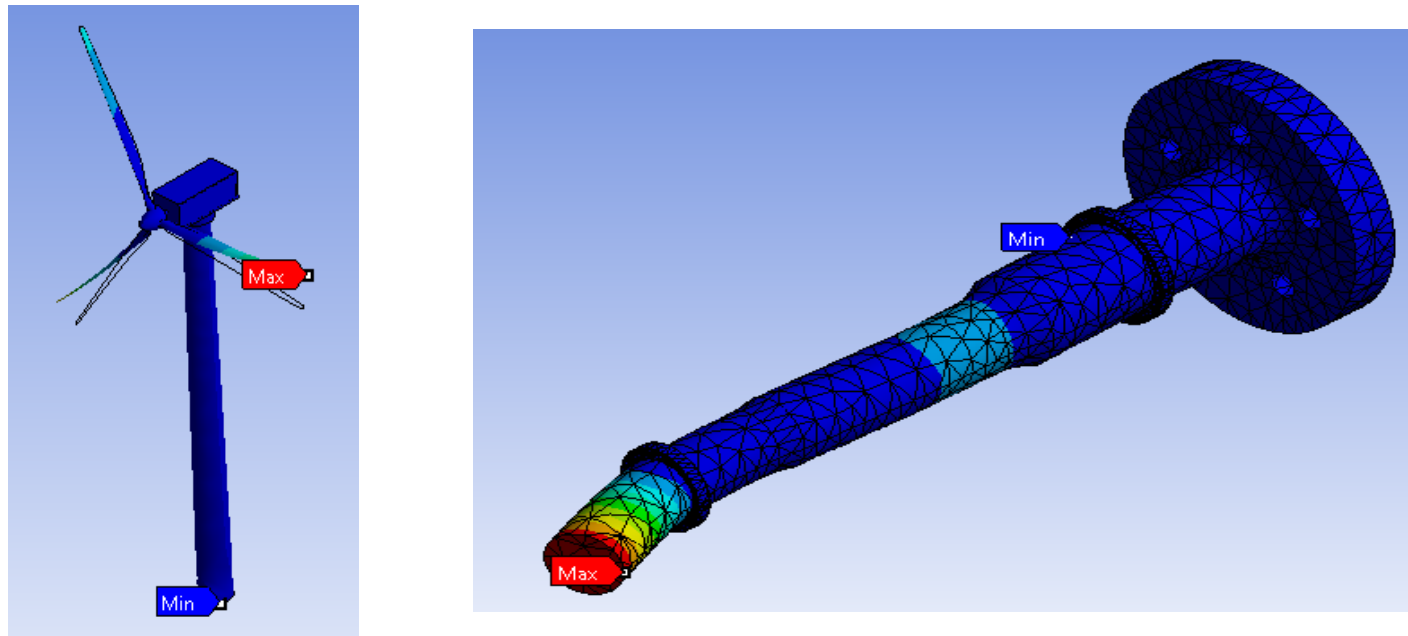


\section{A2. Two Blade HAWT Natural Frequencies}

Two blade HAWT

1

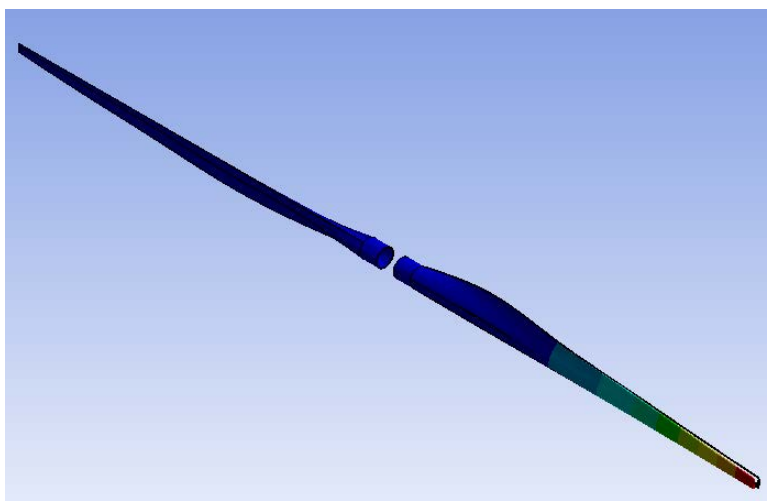

2

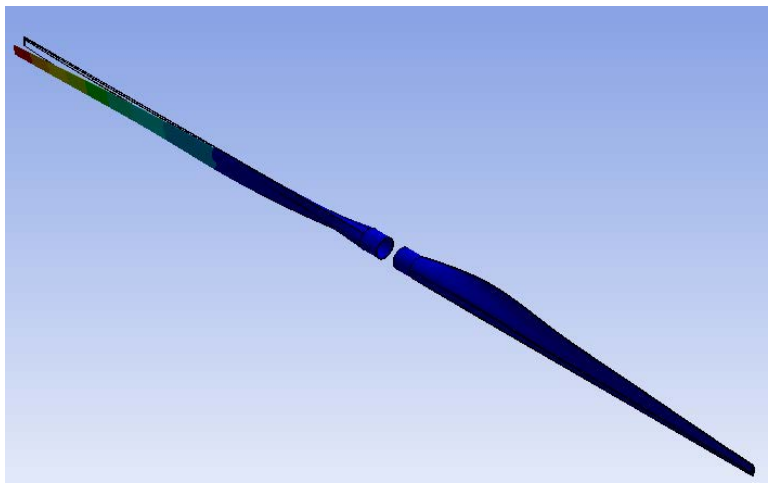

3

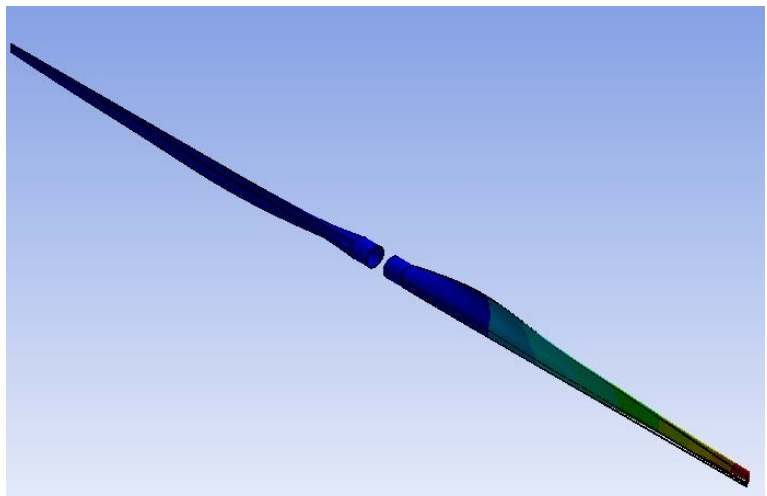

4

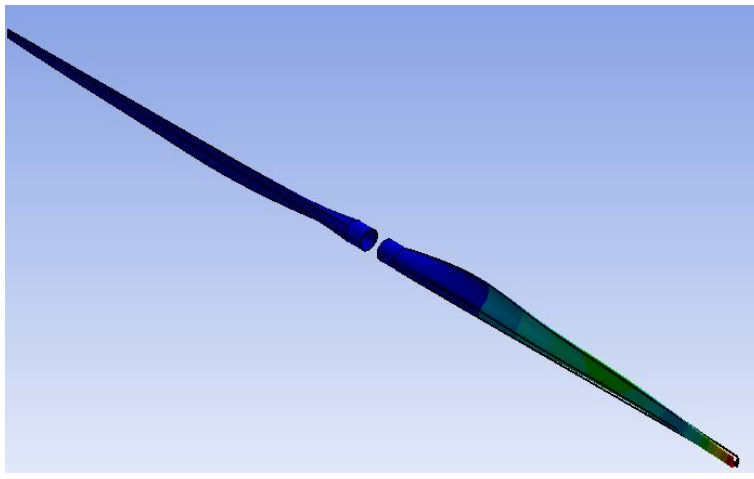




\section{Continued}

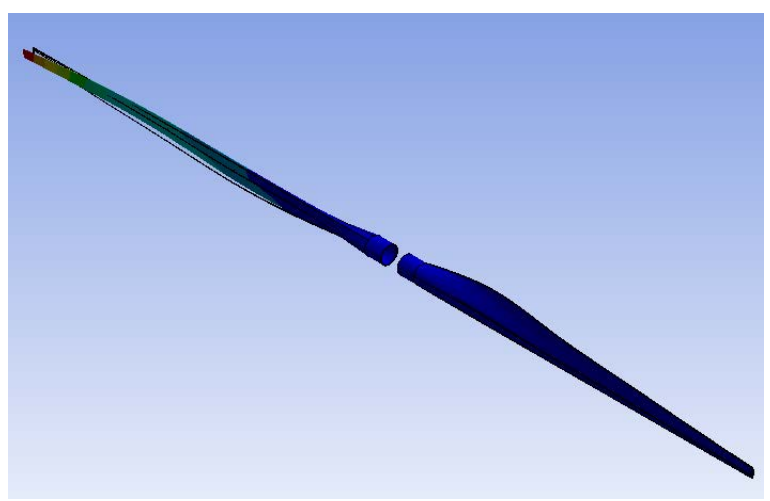

6

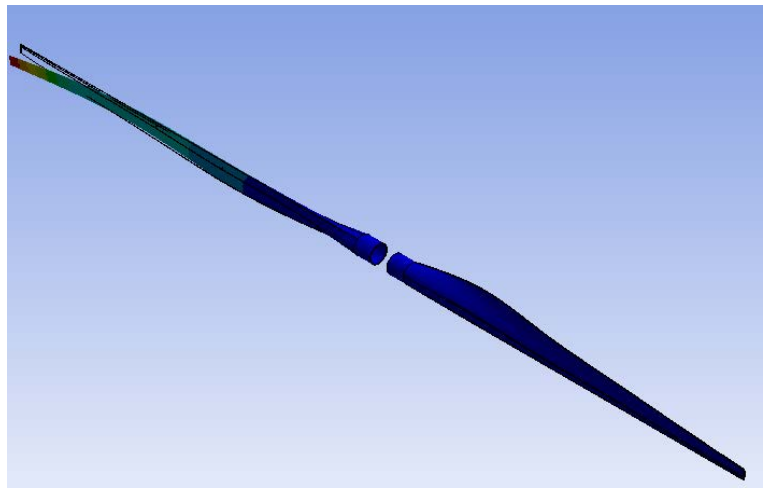

